\title{
Asset market equilibria in cryptocurrency markets: Evidence from a study of privacy and non-privacy coins
}

\author{
Niranjan Sapkota ${ }^{*}, 1$, Klaus Grobys ${ }^{1}$ \\ University of Vaasa, Vaasa, Finland
}

\section{A R T I C L E I N F O}

\section{JEL Classification:}

G12

G14

Keywords:

Cryptocurrency

Bitcoin

Privacy coins

Non-privacy coins

Market equilibria

\begin{abstract}
A B S T R A C T
This paper explores whether asset market equilibria in cryptocurrency markets do exist. In doing so, it distinguishes between privacy and non-privacy coins. Most recently, privacy coins have attracted increasing attention in the public debate as non-privacy cryptocurrencies, such as Bitcoin, do not satisfy some users' demands for anonymity. Analyzing ten cryptocurrencies with the highest market capitalization in each submarket in the 2016-2018 periods, we find that privacy coins exhibit a distinct market equilibrium. Contributing to the current debate on the market efficiency of cryptocurrency markets, our findings provide evidence of market inefficiency. Moreover, the asset market equilibrium of privacy coins appears to originate from non-privacy coins with highest market capitalizations. We argue that the reason for this finding could be that non-privacy coins may be the first choice for criminals who might prefer cryptocurrencies exhibiting both a high level of anonymity and liquidity.
\end{abstract}

\section{Introduction}

Recently, empirical investigations of cryptocurrency markets have attracted considerable attention in the academic literature. This is not surprising given that from an economic perspective the sums of money involved are substantial (Fry and Cheah, 2016, p.350). Bitcoin (BTC), Ethereum (ETH), and Ripple (XRP) are among the top three cryptocurrencies with the highest market capitalization ${ }^{2}$. Interestingly, in recent years, another type of cryptocurrency, exemplified by Monero (XMR), Dash (DASH), and Verge (XVG) came into existence claiming to provide transaction privacy and account balance privacy to their users that the public blockchains like BTC, ETH or XRP do not provide. Androulaki, Karame, Roeschlin, Scherer, and Capkun (2013) argue that almost 40\% of Bitcoin users could be identified despite the privacy measures in place; today, however, virtually all Bitcoin users could be identified. Goldfeder, Kalodner,

\footnotetext{
* Corresponding author at: Department of Accounting and Finance, University of Vaasa, Wolffintie 34, 65200 Vaasa, Finland.

E-mail addresses: niranjan.sapkota@uva.fi (N. Sapkota), klaus.grobys@uva.fi (K. Grobys).

${ }^{1}$ We would like to thank the editor Jonathan Batten and an anonymous reviewer for giving us very helpful and very insightful comments and advice. We thank the participants of the 3rd Cryptocurrency Research Conference 2020, organized by the Centre for Digital Finance, the University of Southampton and the ICMA Centre, for their valuable comments. We are also thankful to the participants of the FINANCE PROPERTY, TECHNOLOGY AND THE ECONOMY CONFERENCE, 2019, University of South Australia, Adelaide, Australia. We also received valuable comments from the participants of the Graduate School Seminar 2018-2019 and Blockchain Seminar 2019, University of Vaasa, Finland.

2 https://coinmarketcap.com/, accessed on 18.3.2019
} 
Reisman, and Narayanan (2018) show how third-party web trackers can de-anonymize users of cryptocurrencies. ${ }^{3}$ By implementing sophisticated cryptographic protocols, privacy coins can hide senders and receivers addresses as well as the transaction amount while executing each transaction. The complete financial transparency of Bitcoin and other non-privacy coins is deterring many institutional and private investors from adopting these decentralized cryptocurrencies (Liu, Li, Karame and Asokan, 2018). The website Bitinfocharts provides user level, account balance level and transaction level information for the top 100 richest wallet addresses of fourteen different cryptocurrencies ${ }^{4}$. Non-privacy coins like Bitcoin are fully transparent; therefore, institutions are hesitant to use it as a medium of exchange. Baur, Hong, and Lee (2018) show that Bitcoins are mainly used as a speculative asset rather than as a medium of exchange.

An alternative is presented by Brenig, Accorsi, and Müller (2015) who outline the structure of a money laundering process and antimoney-laundering controls to examine whether cryptocurrencies constitute a driver for money laundering or not. The study argues that privacy coins have potential benefits for criminals; an important yet neglected factor in the circulation of cryptocurrencies as a money laundering instrument. In this regard, Kethineni and Cao (2020) support Brenig et al. (2015) and argue that cryptocurrencies became the currency of choice for many drug dealers and extortionists because of the opportunities to hide behind the assumed privacy and anonymity.

Privacy coins also use the decentralized public blockchain, but by using many features like masternode technology, ring signature, and a stealth wallet address, privacy coins make it impossible for third parties to trace transactions to the real parties involved. Privacy coins are different from non-privacy coins not only in the cryptographic level, but probably also at the user level. One reason why a separate user base for privacy coins might be growing could be due to the complete transparency of non-privacy coins like BTC and ETH. A certain degree of financial privacy about transaction and balance may be important for both the institutional and individual users. Therefore, one can hypothesize that the traders who favor privacy over complete transparency are emerging as a different subgroup in digital financial markets. Since the total supply of cryptocurrencies is (in most cases) predetermined, the price processes of cryptocurrencies depend solely on the demand side (e.g., the users). As a consequence, if the user base for privacy coins is different from that for non-privacy coins, we would expect that each submarket of cryptocurrencies potentially forms an individual asset market equilibrium.

Hence, we hypothesize that privacy coins form a distinct submarket in the cryptocurrency market. Following Urquhart (2016), Dyhrberg (2016), Bouri, Molnár, Azzi, Roubaud, and Hagfors (2017), who adopt the perspective that cryptocurrencies are an asset market, we consider the privacy and non-privacy coin markets to be two different asset markets. We explore whether market equilibria in cryptocurrency markets exist which, in turn, would imply the existence of submarkets. In this regard, we explicitly test whether privacy coins form such a distinct submarket in the cryptocurrency market. In doing so, we also investigate the efficiency of the overall cryptocurrency market. A test for market efficiency that does not require the specific formulation of an equilibrium price mechanism goes back to an argument by Granger (1986): If two or more asset prices show a stable common relationship in the long-run, it is possible that the movement of one asset price is linked to the movement of other asset prices. In turn, the price of one asset does not only depend on its own past prices but also on the history of a different asset's prices. As a consequence, the weak-form of market efficiency is violated (Richards, 1995, p.632). In line with Engle and Granger's (1987) cointegration theory, we employ Johansen's (1991, 1992a, 1992b, 1994, 1995) multivariate methodology to model and test the long-term equilibrium process. Specifically, if the stable long-run relationship between asset prices is known to the market participants they are able to exploit them to make excess profits (Copeland, 1991, p. 187).

This paper contributes to the literature in several important ways. On the one hand, Urquhart (2016) and Al-Yahyaee, Mensi, and Yoon (2018) studied the market efficiency of Bitcoin and found the cryptocurrency to be inefficient; however, Nadarajah and Chu (2017) revisited Urquhart's (2016) paper and found that Bitcoin returns do satisfy the efficient market hypothesis. Moreover, VidalTomás and Ibañez (2018) and Sensoy (2019) argue that Bitcoin has become more efficient over time, whereas Bariviera's (2017) findings suggest Bitcoin has met the standards of informational efficiency since 2014. The different views in the literature indicate there is no consensus on the market efficiency of cryptocurrencies. While the past papers cited above consider a single asset (e.g., Bitcoin) our paper adds to this strand of literature by taking a market-wide perspective. In doing so, we consider a whole set of cryptocurrencies that exhibit the largest market capitalization and employ Johansen's (1991, 1992a, 1992b, 1994, 1995) multivariate cointegration methodology to explore whether or not asset market equilibria in line with Engle and Granger's (1987) cointegration theory exist.

There is also a new strand of literature emerging that discusses the features of privacy and non-privacy coins. This literature however mostly adopts a technological perspective and explores the privacy implications of Bitcoin (Androulaki et al., 2013), identification of a particular user's blockchain transactions (Goldfeder et al., 2018, Khalilov and Levi 2018), technological interventions that could address the privacy issues of cryptocurrencies (Ouaddah, Elkalam, and Ouahman, 2017; Kopp, Mödinger, Hauck, Kargl, and Bösch, 2017), and potential failures to guarantee privacy in terms of unlinkability and untraceability (Kumar, Fischer, Tople, and Saxena, 2017; Möser et al., 2017). Inspired by Fry and Cheah (2016) and Osterrieder and Lorenz (2017) who discuss the need for academic research on cryptocurrency from a financial point of view, our paper adopts the financial perspective and considers the cryptocurrency market as an asset market comprising two submarkets, the privacy coin market and the non-privacy coin market. This is the first paper that explicitly explores the existence of cointegration relationships among asset prices in the cryptocurrency market.

Finally, there is a wide strand of literature performing market efficiency tests using cointegration analysis of traditional currencies.

\footnotetext{
${ }^{3}$ Third parties typically receive user information for advertising purposes even if users pay via cryptocurrencies, which is enough to identify that particular user's blockchain transactions. By linking the user's cookie to that particular blockchain transaction, one can identify the real user behind it.

${ }^{4}$ https://bitinfocharts.com, accessed on 18.3.2019
} 
Studies that also employ multivariate cointegration analysis as a methodological framework for exploring the European Monetary System (EMS) are Norrbin (1996), Woo (1999), Haug, MacKinnon, and Michels (2000), Rangvid and Sørensen (2002), and Aroskar, Sarkar, and Swanson (2004). Interestingly, those studies are mostly able to reject the null hypothesis of no cointegration for the EMS currencies. No paper has explicitly examined cryptocurrency markets for cointegration. ${ }^{5}$ Hence, our paper extends the literature to reveal potential cointegration equilibria in new digital currency markets.

Our results show strong evidence for the existence of four cointegration relationships in the market for cryptocurrencies. Our evidence suggests that the privacy coin market forms a distinct asset market equilibrium. Employing ten large cap cryptocurrencies with privacy function and ten non-privacy cryptocurrencies in a joint model, we use a fully-specified Vector-Error-Correction Model (VECM) to estimate the four distinct cointegration equilibria. To do so, we employ from both groups of cryptocurrencies (e.g., privacy and non-privacy coins) those cryptocurrencies that exhibit the respective highest and lowest market capitalizations as left-hand side variables. Using Dash (DASH) as the privacy coin with highest market capitalization, we find that only two non-privacy coins, Peercoin (PPC) and MaidSafeCoin (MAID), exhibit $t$-statistics indicating statistical significance on a common $5 \%$ level.

On the other hand, six out of eight privacy coins exhibit $t$-statistics indicating statistical significance on at least a $5 \%$ level, which strongly suggests that this distinct market equilibrium is primarily driven by privacy coins. A joint test for exploring whether or not non-privacy coins are a part of that cointegration relationship shows that we cannot reject the null hypothesis, implying that DASH is a part of a submarket equilibrium consisting of privacy coins that is statistically unrelated to non-privacy coins. Our results are in line with the literature offering evidence of cointegration equilibria in traditional currency markets such as the European Monetary System (Norrbin, 1996; Woo, 1999; Haug et al., 2000; Rangvid and Sørensen, 2002; Aroskar, Sarkar, and Swanson, 2004). The presence of cointegration equilibria is also in line with Urquhart (2016) and Al-Yahyaee, Mensi, and Yoon (2018), who argue that Bitcoin is inefficient because a cointegration relationship implies weak-form market inefficiency. Finally, our results provide some new evidence on market heterogeneity: Privacy coins with high market capitalizations appear to build a submarket of cryptocurrencies as they form their own market equilibrium that is unrelated to the remaining cointegration equilibria in the cryptocurrency market. This market heterogeneity phenomenon in the cryptocurrency market may be subject to future investigations.

\section{Background}

Alexander and Dimitriu (2005) investigate the performance of a cointegration-based index tracking strategy and, by this, test the Efficient Market Hypothesis (EMH), as according to Jensen's (1978) definition of efficient markets, a trading strategy producing significant risk-adjusted payoffs is evidence against the EMH. In this regard, Alexander and Dimitriu (2005, p.215) argue that "the rationale for constructing portfolios based on a cointegration relationship with the benchmark, rather than correlation, rests on the following features of cointegration: the price difference between the benchmark and the replica portfolio is, by construction, stationary; the stock weights, being based on a large amount of history, have an enhanced stability; finally, there is a full use of the information contained in level variables such as stock prices”. As pointed out in Alexander and Dimitriu (2005), assets in price levels contain a higher level of information as opposed to returns. Note that a cointegration relationship is based on an 'historical equilibrium', that is, a price equilibrium estimated from a long historical sample. In this cointegration equilibrium, exogenous cryptocurrencies and endogenous cryptocurrencies share a common stochastic trend that is stationary and mean-reverting. Whereas exogenous cryptocurrencies follow their own stochastic evolutions, endogenous cryptocurrencies adjust to deviations from the longterm equilibrium condition as defined by the cointegration vector. A cointegration relationship is evidence against the EMH because traders could gain profits by exploiting the mean-reverting characteristic defined by a cointegration relationship. In this regard, we follow Alexander and Dimitriu (2005) and test the EMH in the market for cryptocurrencies. In doing so, our paper contributes to the literature on testing the market efficiency of cryptocurrency markets using cointegration theory (Engle and Granger's, 1987).

Furthermore, Borri's (2019) study indicates that cryptocurrency returns are highly correlated one with the other. However, she finds that using cryptocurrency portfolios may substantially reduce idiosyncratic risk and may offer better risk-adjusted and conditional returns than individual cryptocurrencies. In this regard, it is interesting to note that Borri (2019) does not study cryptocurrency portfolios that form submarkets within the overall cryptocurrency ecosystem. Using popular moving average strategies, a recent study from Ahmed, Grobys, Sapkota (2020) explores the profitability of technical trading rules implemented among cryptocurrencies with privacy function. Averaging the average returns across the entire set of cryptocurrencies with privacy function, they did not find any positive average portfolio returns in excess of the equally-weighted average buy-and-hold portfolio. This is a surprising finding because earlier literature suggests that technical trading rules are profitable for cryptocurrency markets (Grobys et al., 2020; Gerritsen et al., 2020; Corbet et al., 2019; Miller et al., 2019). Ahmed et al.'s (2020) study does not include any fully elaborated dynamic general equilibrium asset-pricing models to assess whether the reported payoffs are merely the equilibrium rents that accrue to investors willing to carry the risks associated with such strategies (Lo, Mamaysky, and Wang, 2000) and, hence, encourages studies to discern the economic sources of return differentials among cryptocurrency submarkets. Our study contributes to this strand of literature by taking an important next step by exploring whether statistical equilibriums in cryptocurrency submarkets do exist.

Moreover, we note that Ahmed et al.'s (2020) study provides some evidence for that the market forces driving the market for cryptocurrencies with privacy function are somewhat different from those that drive the overall cryptocurrency market. It is interesting to note that recent research suggests that about half of Bitcoin transactions is associated with criminal activities such as drugs,

\footnotetext{
${ }^{5}$ Other relevant papers that document mixed results are Jeon and Seo (2003) and Phengpis (2006), who examine structural instability in particular and investigate currency crises for market efficiency.
} 
Table 1

Top 10 Privacy and Non-privacy Coins.

\begin{tabular}{|c|c|c|c|c|c|c|c|}
\hline S.No & $\begin{array}{l}\text { Non-Privacy } \\
\text { Coin }\end{array}$ & Symbol & $\begin{array}{l}\text { January } 3, \\
2016 \text { Coin Rank } \\
\text { /572 Coins }\end{array}$ & $\begin{array}{l}\text { Capitalization } \\
\text { (\$) }\end{array}$ & $\begin{array}{l}\text { December 30, } \\
2018 \text { Coin } \\
\text { Rank/2073 } \\
\text { Coins }\end{array}$ & $\begin{array}{l}\text { Capitalization } \\
\text { (\$) }\end{array}$ & $\begin{array}{l}3 \text { Years' Market } \\
\text { Capitalization } \\
\text { Growth\% }\end{array}$ \\
\hline & Bitcoin & BTC & 1 & $6,467,437,080$ & 1 & $67,475,512,827$ & 943.31 \\
\hline 1 & Ripple & XRP & 2 & $201,799,631$ & 2 & $15,076,740,856$ & 7371.14 \\
\hline 2 & Litecoin & LTC & 3 & $152,873,521$ & 7 & $1,912,263,648$ & 1150.90 \\
\hline 3 & Ethereum & ETH & 4 & $73,843,278$ & 3 & $14,560,066,114$ & 19617.52 \\
\hline 4 & Dogecoin & DOGE & 6 & $14,940,681$ & 23 & $278,470,304$ & 1763.84 \\
\hline 5 & Peercoin & PPC & 7 & $9,756,959$ & 181 & $14,254,314$ & 46.10 \\
\hline 6 & BitShares & BTS & 8 & $8,591,688$ & 44 & $106,520,276$ & 1139.81 \\
\hline 7 & Stellar & XLM & 9 & $8,436,465$ & 6 & $2,250,048,215$ & 26570.51 \\
\hline 8 & Nxt & NXT & 10 & $6,863,998$ & 113 & $29,426,627$ & 328.71 \\
\hline 9 & MaidSafeCoin & MAID & 11 & $6,789,470$ & 65 & $61,335,019$ & 803.38 \\
\hline \multirow[t]{2}{*}{10} & NameCoin & NMC & 12 & $6,073,338$ & 220 & $10,480,336$ & 72.56 \\
\hline & & & & & & $\begin{array}{l}\text { Average } \\
\text { (Excluding } \\
\text { Bitcoin) }\end{array}$ & 5886.45 \\
\hline \multicolumn{8}{|c|}{ Panel B: Top 10 Privacy Coins } \\
\hline S.No & $\begin{array}{l}\text { Privacy Coin } \\
\text { Name }\end{array}$ & & & & & & \\
\hline 1 & Dash & DASH & 5 & $19,794,713$ & 15 & $698,091,183$ & 3426.65 \\
\hline 2 & Bytecoin & BCN & 14 & $5,582,979$ & 38 & $134,517,567$ & 2309.42 \\
\hline 3 & Monero & XMR & 15 & $5,295,952$ & 13 & $806,939,516$ & 15136.91 \\
\hline 4 & DigitalNote & XDN & 51 & 447,057 & 252 & $8,565,009$ & 1815.87 \\
\hline 5 & CloakCoin & CLOAK & 125 & 201,995 & 318 & $6,249,733$ & 2994.00 \\
\hline 6 & Aeon & AEON & 137 & 137,088 & 393 & $4,475,790$ & 3164.90 \\
\hline 7 & NavCoin & NAV & 142 & 121,805 & 213 & $11,127,542$ & 9035.54 \\
\hline 8 & Verge & XVG & 149 & 109,968 & 43 & $110,307,808$ & 100209.00 \\
\hline 9 & Stealth & XST & 161 & 8352 & 515 & $2,539,074$ & 30300.79 \\
\hline \multirow[t]{2}{*}{10} & Prime-XI & PXI & 322 & 8889 & 1701 & 4236 & -52.35 \\
\hline & & & & & & Average & 16834.07 \\
\hline
\end{tabular}

Note: This table reports the top 11 non-privacy coins (including Bitcoin) and top ten privacy coins based on their market capitalization as of January 3 , 2016. There were 572 cryptocurrencies available (including both privacy and non-privacy coins) as of January 3, 2016, and 2073 coins as of December 30, 2018. Coin Rank shows the position based on a coin's market capitalization. Three Years' Market

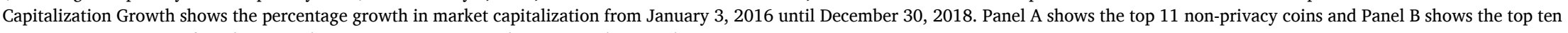
privacy coins in terms of market capitalization (Source: coinmarketcap.com/historical/s. 
Table 2

ADF tests for privacy and non-privacy coins.

\begin{tabular}{|c|c|c|c|c|c|c|c|c|c|}
\hline \multicolumn{5}{|c|}{ Privacy coins } & \multicolumn{5}{|c|}{ Non-privacy coins } \\
\hline \multirow[b]{2}{*}{ Coin } & \multicolumn{2}{|l|}{ Model 1} & \multicolumn{2}{|l|}{ Model 2} & \multirow[b]{2}{*}{ Coin } & \multicolumn{2}{|l|}{ Model 1} & \multicolumn{2}{|l|}{ Model 2} \\
\hline & Intercep $^{\mathrm{a}}$ & $\operatorname{Lags}^{\mathrm{d}}$ & Intercept and trend ${ }^{b}$ & Lags $^{d}$ & & Intercept $^{\mathrm{a}}$ & Lags $^{d}$ & Intercept and trend ${ }^{b}$ & Lags $^{d}$ \\
\hline DASH & -1.79 & 0 & 0.25 & 0 & XRP & -0.99 & 2 & -1.19 & 2 \\
\hline BCN & -1.34 & 1 & -0.90 & 1 & LTC & -1.13 & 0 & -0.23 & 0 \\
\hline XDN & -1.39 & 0 & -0.91 & 0 & ETH & $-2.93^{* *}$ & 0 & -0.63 & 0 \\
\hline XMR & -2.16 & 0 & -0.15 & 0 & DOGE & -1.30 & 0 & -1.41 & 0 \\
\hline CLOAK & -1.51 & 2 & -0.72 & 2 & PPC & -1.43 & 0 & -1.18 & 0 \\
\hline AEON & -1.46 & 1 & -0.57 & 1 & BTS & -1.24 & 0 & -0.41 & 0 \\
\hline XST & -1.49 & 1 & -1.45 & 1 & XLM & -0.93 & 1 & -1.45 & 1 \\
\hline PXI & -1.44 & 2 & -1.16 & 2 & NXT & -1.37 & 0 & -0.62 & 0 \\
\hline NAV & -1.86 & 4 & -0.60 & 4 & MAID & $-2.86^{* *}$ & 0 & -1.69 & 1 \\
\hline \multirow[t]{2}{*}{ XVG } & -1.23 & 4 & -1.27 & 4 & NMC & -1.65 & 1 & -1.59 & 1 \\
\hline & & & & & BTC & -1.31 & 0 & -0.06 & 0 \\
\hline
\end{tabular}

Note: This table reports the results for Augmented Dickey Fuller tests of the daily price series in logs for privacy and non-privacy coins. Model 1 accounts for an intercept in the test regression, whereas model 2 accounts for both an intercept and trend term. The sample period is from January 1 , 2016 until December 31, 2018 corresponding to 1096 observations.

$* *$ Statistically significant on a $5 \%$ level.

${ }^{\mathrm{a} C r i t i c a l}$ values for $10 \%, 5 \%$ and $1 \%$ significance levels are $-2.57,-2.86$ and -3.44 .

${ }^{\mathrm{b}}$ Critical values for $10 \%, 5 \%$ and $1 \%$ significance levels are $-3.13,-3.41$ and -3.97 .

${ }^{\mathrm{d}}$ Lag-order is chosen by using the Schwarz info criterion. The maximum lag length is chosen by default is 21 .

for instance (Foley, Karlsen, Putniņš, 2019). Because Bitcoin is a non-privacy coin, Ahmed et al. (2020) argue that the only option how market participants might achieve (full) anonymity is via the dark web. On the other hand, the usage of the dark web is per se a criminal offence; as a consequence, traders might prefer choosing privacy coins for their transactions instead of non-privacy coins. "This enables users making transactions in cryptocurrency (e.g., privacy coins) in the legal world-wide-web domain while still meeting their demands for legal transfers of digital currency, security, and confidentiality through anonymous transactions. Moreover, such security features may be of considerable importance for traders from countries where economic and political freedom is limited." (Ahmed et al., 2020). Since the price of cryptocurrencies is in most cases (due to the limited supply) driven by the demand side, we hypothesize in line with Ahmed et al. (2020) that market participants engaging in criminal activities shift their demand from nonprivacy to privacy cryptocurrencies. If those market participants constituted a distinct user base it would imply that a potential market equilibrium in the submarket for cryptocurrencies exhibiting the privacy function should consequently be detached from the market equilibrium formed by non-privacy cryptocurrencies. Motivated by this literature, we take a novel perspective using cointegration theory to explore whether distinct market equilibria in those two different cryptocurrency submarkets do exist.

\section{Methodology}

For each group — privacy and non-privacy coins —-we retrieved daily closing prices ${ }^{6}$ for ten cryptocurrencies that exhibit the highest market capitalization as of January 3, 2016. We also downloaded data for Bitcoin, which dominates the cryptocurrency market. Our sample is from January 1, 2016 until December 31, 2018 accounting for 1096 daily observations. For all asset prices we compound the log-price series that we used in the following analyses. The trend in data levels of non-privacy and privacy coins are reported in Fig. A1 and Fig. A2 in the appendix.

Table 1 illustrates that at the beginning of our sample period, the average market capitalization of non-privacy coins (excluding Bitcoin) is about 19 times larger than that of privacy coins. Interestingly, the three-year growth in market capitalization of these top ten cryptocurrencies in each category is about three times higher for privacy coins than for non-privacy coins. This suggests that the relative popularity of privacy coins has increased over time. Even though both privacy and non-privacy categories of cryptocurrencies use the decentralized public blockchain technology, they differ on other technological levels, particularly in relation to either the public node, wallet form, or the signature. Privacy coins also differ from non-privacy coins in terms of their usage. People who use nonprivacy coins face complete financial transparency which might be less appealing in a competitive environment. Traders might want to maintain a certain degree of financial privacy at both the transaction and account balance level. Thus, we hypothesize that, as independent players emerge in digital currency markets, privacy coins form a distinct submarket.

To investigate whether or not distinct market equilibria, forming submarkets in the overall cryptocurrency market, exist and to explore how they relate to each other, the first step of our empirical analysis is to identify whether or not our set of cryptocurrencies exhibits stationarity. This is an important step because cointegration equilibria require that the input variables are integrated of order one, that is, $I(1)$ stochastic processes. To test the order of integration, we follow the common literature and employ the well-known Augmented Dickey Fuller (ADF) unit root test (Dickey-Fuller, 1979). The model for estimating the test statistics is given by:

\footnotetext{
${ }^{6}$ Because cryptocurrencies are traded 24/7, coinmarketcap.com takes the latest data in the range (UTC time) to determine the closing price.
} 
Table 3

Trace test excluding Bitcoin.

\begin{tabular}{|c|c|c|c|c|c|}
\hline Rank & Eigenvalue & Trace test & p-value & Lmax test & $\mathrm{p}$-value \\
\hline 0 & 0.1390 & 1094.7000 & {$[0.0000]$} & 163.7500 & {$[0.0000]$} \\
\hline 1 & 0.1227 & 931.0000 & {$[0.0000]$} & 143.2300 & {$[0.0001]$} \\
\hline 2 & 0.0926 & 787.7700 & [0.0043] & 106.3600 & {$[0.2285]$} \\
\hline 3 & 0.0830 & 681.4100 & {$[0.0402]$} & 94.7980 & {$[0.4534]$} \\
\hline 4 & 0.0752 & 586.6200 & [0.1597] & 85.4960 & [0.6187] \\
\hline 5 & 0.0676 & 501.1200 & {$[0.3743]$} & 76.5640 & {$[0.7612]$} \\
\hline 6 & 0.0526 & 424.5500 & {$[0.6062]$} & 59.0730 & [0.9899] \\
\hline 7 & 0.0519 & 365.4800 & {$[0.6468]$} & 58.2950 & {$[0.9560]$} \\
\hline 8 & 0.0491 & 307.1900 & [0.7431] & 55.1160 & [0.9217] \\
\hline 9 & 0.0407 & 252.0700 & [0.8473] & 45.4780 & {$[0.9817]$} \\
\hline 10 & 0.0386 & 206.5900 & {$[0.8753]$} & 43.0240 & [0.9529] \\
\hline 11 & 0.0303 & 163.5700 & [0.9204] & 33.6620 & [0.9921] \\
\hline 12 & 0.0265 & 129.9100 & [0.9093] & 29.4100 & [0.9895] \\
\hline 13 & 0.0243 & 100.5000 & {$[0.8892]$} & 26.9620 & {$[0.9671]$} \\
\hline 14 & 0.0233 & 73.5350 & [0.8887] & 25.8330 & {$[0.8615]$} \\
\hline 15 & 0.0140 & 47.7020 & [0.9404] & 15.4110 & [0.9891] \\
\hline 16 & 0.0130 & 32.2900 & {$[0.8650]$} & 14.2800 & {$[0.9204]$} \\
\hline 17 & 0.0104 & 18.0110 & [0.8192] & 11.4910 & {$[0.8060]$} \\
\hline 18 & 0.0052 & 6.5194 & {$[0.8243]$} & 5.7163 & {$[0.8390]$} \\
\hline 19 & 0.0007 & 0.8031 & {$[0.3702]$} & 0.8031 & {$[0.3702]$} \\
\hline
\end{tabular}

Note: This table reports the results for the trace test for cointegration applied to a set of twenty cryptocurrencies consisting of ten privacy coins and ten non-privacy coins (excluding Bitcoin) exhibiting the highest market capitalization as of Jan 3, 2016. Our model uses daily data of log prices. The test statistic allows for linear deterministic trend in data. The model is estimated with the econometric software Gretl. The sample period is from January 1, 2016 until December 31, 2018 corresponding to 1096 observations.

Johansen test:

Number of equations $=20$

Lag order $=2$

Estimation period: 2016-01-03-2018-12-31 $(\mathrm{T}=1094)$

Case 5: Unrestricted trend and constant

Log-likelihood $=45917.6$ (including constant term: 42813)

$$
\Delta p_{i, t}=\delta_{0, i}+\delta_{1, i} t+\delta_{2, i} p_{i, t-1}+\sum_{j=1}^{T} \delta_{2+j, i} \Delta p_{i, t-j}+\varepsilon_{i, t}
$$

where $p_{i, t}$ denotes the $\log$-price of cryptocurrency $i$ at time $t, \delta_{0}$ is the corresponding estimate for the intercept of the regression, $\delta_{1}$ is the corresponding estimate for the time trend $t, \delta_{2+j}$ is the estimate for the lagged differences in $\log$ prices of lag $2+j$ where $j=1, \cdots, T, \varepsilon_{t}$ denotes a white-noise error term, and the parameter $\delta_{2}$ is assumed to be zero under the null hypothesis. Under the null hypothesis, $p_{i, t}$ is an $I(1)$ stochastic process. Given our research context, we assume stationarity under the alternative hypothesis. Moreover, we follow a common practice by choosing the lag-order $j$ with respect to the Schwarz criterion.

Specifically, under some circumstances, a linear combination of some $I(1)$ processes becomes a stationary process, that is, an $I(0)$ process. According to Granger (1986), and Engle and Granger (1987), those $k=1, \cdots, K$ stochastic processes are said to be cointegrated of order $(1, \ldots, 1)$, denoted as $C I(1, \ldots, 1)$. To test the order of cointegration, we employ the trace test using the overall set of $K=20$ cryptocurrencies, consisting of ten privacy coins and ten non-privacy coins, given by:

$$
L R\left(r_{0}\right)=-T \sum_{j=r_{0}+1}^{K} \log \left(1-\lambda_{j}\right)
$$

where $\lambda_{j}$ are the eigenvalues obtained by applying Reduced Rank (RR) regression techniques to the fully unrestricted Vector-Error Correction model (VECM) (Johansen 1991, 1992a, 1992b, 1994, 1995) given by:

$$
\Delta \boldsymbol{y}_{t}-\mu_{1}=\Pi\left(\boldsymbol{y}_{t-1}-\mu_{0}-\mu_{1}(t-1)\right)+\sum_{j=1}^{p-1} \Gamma_{j}\left(\Delta \boldsymbol{y}_{t-1}-\mu_{1}\right)+\boldsymbol{u}_{t}
$$

where the $20 \mathrm{x} 1$ vector $\Delta \boldsymbol{y}_{t}$ contains the log-returns of cryptocurrencies $i=\{1,2, \cdots, 19,20\} .{ }^{7}$ Moreover, $\mu_{0}$ and $\mu_{1}$ denote constant and trend, $\Pi$ and $\Gamma_{1}, \Gamma_{2}, \ldots, \Gamma_{p-1}$ are $K x K$ parameter matrices. The trace test tests the sequence of hypotheses given by:

\footnotetext{
7 The model is more detailed in Lütkepohl, Krätzig, and Phillips (2004, p.114).
} 
Table 4

Trace test including Bitcoin.

\begin{tabular}{|c|c|c|c|c|c|}
\hline Rank & Eigenvalue & Trace test & p-value & Lmax test & p-value \\
\hline 0 & 0.1408 & 1196.4000 & {$[0.0000]$} & 166.0300 & [0.0000] \\
\hline 1 & 0.1268 & 1030.4000 & {$[0.0000]$} & 148.3400 & [0.0002] \\
\hline 2 & 0.1057 & 882.0600 & {$[0.0015]$} & 122.1900 & {$[0.0407]$} \\
\hline 3 & 0.0873 & 759.8800 & [0.0344] & 99.9500 & {$[0.4744]$} \\
\hline 4 & 0.0821 & 659.9300 & {$[0.1408]$} & 93.7200 & [0.5010] \\
\hline 5 & 0.0693 & 566.2100 & {$[0.3807]$} & 78.5400 & {$[0.8712]$} \\
\hline 6 & 0.0632 & 487.6700 & [0.5692] & 71.4400 & [0.9119] \\
\hline 7 & 0.0520 & 416.2300 & [0.7268] & 58.4500 & [0.9921] \\
\hline 8 & 0.0498 & 357.7800 & {$[0.7611]$} & 55.9000 & [0.9797] \\
\hline 9 & 0.0422 & 301.8800 & {$[0.8170]$} & 47.1600 & [0.9955] \\
\hline 10 & 0.0393 & 254.7300 & [0.8125] & 43.8500 & [0.9906] \\
\hline 11 & 0.0384 & 210.8800 & [0.8174] & 42.8300 & [0.9558] \\
\hline 12 & 0.0332 & 168.0500 & [0.8682] & 36.9300 & [0.9671] \\
\hline 13 & 0.0256 & 131.1200 & [0.8939] & 28.3400 & [0.9940] \\
\hline 14 & 0.0245 & 102.7900 & [0.8492] & 27.0900 & [0.9653] \\
\hline 15 & 0.0239 & 75.7000 & [0.8440] & 26.5700 & [0.8288] \\
\hline 16 & 0.0170 & 49.1400 & [0.9169] & 18.7900 & [0.9322] \\
\hline 17 & 0.0126 & 30.3400 & [0.9183] & 13.9100 & [0.9331] \\
\hline 18 & 0.0092 & 16.4300 & [0.8896] & 10.0300 & [0.8961] \\
\hline 19 & 0.0050 & 6.4000 & {$[0.8346]$} & 5.5400 & {$[0.8541]$} \\
\hline 20 & 0.0008 & 0.8600 & [0.3548] & 0.8600 & {$[0.3548]$} \\
\hline
\end{tabular}

Note: This table reports the results for the trace test for cointegration applied to a set of twenty-one cryptocurrencies consisting ten privacy coins and eleven non-privacy coins (including Bitcoin) exhibiting the highest market capitalization as of Jan 3, 2016. Our model uses daily data of log prices. The test statistic allows for linear deterministic trend in data. The model is estimated with the econometric software Gretl. The sample period is from January 1, 2016 until December 31, 2018 corresponding to 1096 observations.

Johansen test:

Number of equations $=21$

Lag order $=2$

Estimation period: 2016-01-03-2018-12-31 ( $\mathrm{T}=1094)$

Case 5: Unrestricted trend and constant

Log-likelihood $=49307.7$ (including constant term: 46203.1)

$$
\begin{array}{lll}
H_{0}(0): \operatorname{rank}(\Pi)=0 & \text { versus } & H_{1}(0): \operatorname{rank}(\Pi)>0, \\
H_{0}(1): \operatorname{rank}(\Pi)=1 & \text { versus } & H_{1}(0): \operatorname{rank}(\Pi)>1, \\
& \vdots & \\
H_{0}(K-1): \operatorname{rank}(\Pi)=K-1 & \text { versus } & H_{1}(K-1): \operatorname{rank}(\Pi)=K .
\end{array}
$$

The corresponding cointegration rank is selected when the null hypothesis cannot be rejected for the first time. ${ }^{8}$ If a cointegration relationship of order $\boldsymbol{r}$ exists, the matrix $\Pi$ has a reduced rank form and can be decomposed into $\alpha \boldsymbol{\beta}$ ', where $\boldsymbol{\alpha}$ and $\boldsymbol{\beta}$ are $K \mathrm{x} r$ matrices. In this regard, the term,

$$
\boldsymbol{\beta}^{\prime}\left(\boldsymbol{y}_{t-1}-\mu_{0}-\mu_{1}(t-1)\right)
$$

contains the cointegration equilibrium relationships, whereas $\alpha$ is the loading matrix. Each cryptocurrency in submarket $r$ that has an insignificant weight attached to the respective cointegration relationship is said to be exogenous as it does not respond to disturbances of the long-term equilibrium. If cointegration holds, the linear combination conditioned by the matrix

$\boldsymbol{\beta}$ results in $r$ stationary stochastic processes that are mean-reverting. While exogenous cryptocurrencies entering the respective cointegration equilibrium do not mean-revert, those cryptocurrencies that are endogenous adjust to deviations from the long-term equilibrium defined by $\boldsymbol{\beta}$ (via mean-reversion). Corresponding significant loadings in the matrix $\boldsymbol{\alpha}$ suggest endogeneity. Traders could exploit this pattern by betting on this mean-reversion property. As pointed out in Alexander and Dimitriu (2005), the presence of a cointegration relationship is evidence against the EMH because traders could gain profits by exploiting the mean-reverting characteristic defined by a cointegration relationship.

\section{Results}

The results of testing the order of integration are presented in Table 2. Initially imposing the restriction $\delta_{1, i}=0$, we find that none of the privacy coins appears to be stationary, whereas the null hypothesis is rejected for only two non-privacy coins (e.g., Ethereum and MaidSafeCoin). However, when estimating the fully unrestricted ADF test-involving testing a random walk with drift under the null hypothesis against a trend-stationary process under the alternative-we find that all cryptocurrencies appear to be I(1). This result is in line with the earlier literature indicating that Bitcoin is an I(1) process (Urquhart, 2016).

${ }^{8}$ Using the general-to-specific rule, we made use of the fully unrestricted VECM as given in Eq. (3). 
Table 5

Vector-Error Correction model estimates using all 20 cryptocurrencies excluding Bitcoin.

\begin{tabular}{|c|c|c|c|c|c|c|c|c|c|}
\hline$i$ & Coins & $\widehat{\boldsymbol{\beta}}_{1, i}$ & $\widehat{\boldsymbol{\beta}}_{2, i}$ & $\widehat{\boldsymbol{\beta}}_{3, i}$ & $\widehat{\boldsymbol{\beta}}_{4, i}$ & $\widehat{\boldsymbol{\alpha}}_{1, i}$ & $\widehat{\boldsymbol{\alpha}}_{2, i}$ & $\widehat{\boldsymbol{\alpha}}_{3, i}$ & $\widehat{\boldsymbol{\alpha}}_{4, i}$ \\
\hline 1 & XRP $(-1)$ & $\begin{array}{c}1.0000 \\
(0.0000)\end{array}$ & $\begin{array}{c}0.0000 \\
(0.0000)\end{array}$ & $\begin{array}{c}0.0000 \\
(0.0000)\end{array}$ & $\begin{array}{c}0.0000 \\
(0.0000)\end{array}$ & $\begin{array}{l}-0.0038 \\
(-0.4440)\end{array}$ & $\begin{array}{l}-0.0186^{* * *} \\
(-2.5970)\end{array}$ & $\begin{array}{c}0.0141^{*} \\
(1.6730)\end{array}$ & $\begin{array}{c}0.0020 \\
(0.3506)\end{array}$ \\
\hline 2 & DASH $(-1)$ & $\begin{array}{c}0.0000 \\
(0.0000)\end{array}$ & $\begin{array}{c}1.0000 \\
(0.0000)\end{array}$ & $\begin{array}{c}0.0000 \\
(0.0000)\end{array}$ & $\begin{array}{c}0.0000 \\
(0.0000)\end{array}$ & $\begin{array}{l}0.0111 \\
(1.6430)\end{array}$ & $\begin{array}{l}-0.0005 \\
(-0.0812)\end{array}$ & $\begin{array}{c}-0.0030 \\
(-0.4512)\end{array}$ & $\begin{array}{l}0.0156^{* * *} \\
(3.352)\end{array}$ \\
\hline 3 & NMC(-1) & $\begin{array}{c}0.0000 \\
(0.0000)\end{array}$ & $\begin{array}{c}0.0000 \\
(0.0000)\end{array}$ & $\begin{array}{c}1.0000 \\
(0.0000)\end{array}$ & $\begin{array}{c}0.0000 \\
(0.0000)\end{array}$ & $\begin{array}{l}0.0180 * \\
(1.6900)\end{array}$ & $\begin{array}{l}0.0076 \\
(0.8479)\end{array}$ & $\begin{array}{l}-0.0187^{*} \\
(-1.769)\end{array}$ & $\begin{array}{l}0.0218^{* * *} \\
(2.975)\end{array}$ \\
\hline 4 & $\operatorname{PXI}(-1)$ & $\begin{array}{c}0.0000 \\
(0.0000)\end{array}$ & $\begin{array}{c}0.0000 \\
(0.0000)\end{array}$ & $\begin{array}{c}0.0000 \\
(0.0000)\end{array}$ & $\begin{array}{c}1.0000 \\
(0.0000)\end{array}$ & $\begin{array}{l}-0.0024 \\
(-0.1158)\end{array}$ & $\begin{array}{l}0.0217 \\
(1.2260)\end{array}$ & $\begin{array}{l}0.0657^{* * *} \\
(3.1520)\end{array}$ & $\begin{array}{l}-0.0649^{* * *} \\
(-4.5020)\end{array}$ \\
\hline 5 & $\operatorname{LTC}(-1)$ & $\begin{array}{c}-0.22378 \\
(-0.6291)\end{array}$ & $\begin{array}{c}0.25449 \\
(0.5822)\end{array}$ & $\begin{array}{c}-0.3565^{*} \\
(-1.6127)\end{array}$ & $\begin{array}{l}-1.2630^{* * *} \\
(-3.8480)\end{array}$ & $\begin{array}{l}0.0094 \\
(1.4610)\end{array}$ & $\begin{array}{l}-0.0064 \\
(-1.1890)\end{array}$ & $\begin{array}{c}-0.0048 \\
(-0.7491)\end{array}$ & $\begin{array}{l}0.0193^{* * *} \\
(4.3810)\end{array}$ \\
\hline 6 & $\mathrm{BCN}(-1)$ & $\begin{array}{l}0.6816^{* * *} \\
(2.6323)\end{array}$ & $\begin{array}{c}-0.4986 \\
(-1.5670)\end{array}$ & $\begin{array}{l}-1.0800^{* * *} \\
(-6.7180)\end{array}$ & $\begin{array}{l}-1.2040^{* * *} \\
(-5.0368)\end{array}$ & $\begin{array}{l}0.0498 * * * * \\
(3.9960)\end{array}$ & $\begin{array}{l}0.0241^{* *} \\
(2.2860)\end{array}$ & $\begin{array}{l}0.0660 * * * \\
(5.3260)\end{array}$ & $\begin{array}{c}0.0147^{*} \\
(1.7130)\end{array}$ \\
\hline 7 & ETH(-1) & $\begin{array}{c}0.1403 \\
(0.5667)\end{array}$ & $\begin{array}{c}-0.5074 * \\
(-1.6679)\end{array}$ & $\begin{array}{c}0.1487 \\
(0.9668)\end{array}$ & $\begin{array}{l}0.7983^{* * *} \\
(3.4939)\end{array}$ & $\begin{array}{l}0.0176^{* * *} \\
(2.4650)\end{array}$ & $\begin{array}{l}0.0120^{* *} \\
(2.0000)\end{array}$ & $\begin{array}{l}0.0165^{* *} \\
(2.3310)\end{array}$ & $\begin{array}{c}0.0005 \\
(0.0930)\end{array}$ \\
\hline 8 & XMR(-1) & $\begin{array}{l}2.7010^{* * * *} \\
(7.7472)\end{array}$ & $\begin{array}{l}-2.4470^{* * *} \\
(-5.7110)\end{array}$ & $\begin{array}{l}-1.0580^{* * *} \\
(-4.8846)\end{array}$ & $\begin{array}{l}-2.4440^{* * * *} \\
(-7.5971)\end{array}$ & $\begin{array}{l}0.0253^{* * *} \\
(3.1810)\end{array}$ & $\begin{array}{l}0.0133^{* *} \\
(1.9850)\end{array}$ & $\begin{array}{c}0.0022 \\
(0.2730)\end{array}$ & $\begin{array}{l}0.0132^{* * *} \\
(2.4090)\end{array}$ \\
\hline 9 & DOGE(-1) & $\begin{array}{c}-0.2443 \\
(-0.7941)\end{array}$ & $\begin{array}{c}-0.2423 \\
(-0.6409)\end{array}$ & $\begin{array}{l}-0.4449^{* *} \\
(-2.3270)\end{array}$ & $\begin{array}{l}0.7019^{* *} \\
(2.4723)\end{array}$ & $\begin{array}{l}0.0365^{* * *} \\
(4.7090)\end{array}$ & $\begin{array}{l}0.0127^{*} \\
(1.9390)\end{array}$ & $\begin{array}{l}0.0236 \text { *** } \\
(3.0690)\end{array}$ & $\begin{array}{c}0.0064 \\
(1.2100)\end{array}$ \\
\hline 10 & $\mathrm{XDN}(-1)$ & $\begin{array}{l}-1.3143^{* * *} \\
(-5.429)\end{array}$ & $\begin{array}{l}1.9534^{* * *} \\
(6.5663)\end{array}$ & $\begin{array}{c}-0.0193 \\
(-0.1281)\end{array}$ & $\begin{array}{l}-0.7147^{\text {k** }} \\
(-3.1993)\end{array}$ & $\begin{array}{l}0.0660^{* * *} \\
(5.7470)\end{array}$ & $\begin{array}{l}0.0032 \\
(0.3348)\end{array}$ & $\begin{array}{c}0.0019 \\
(0.1648)\end{array}$ & $\begin{array}{l}0.0466^{* * *} \\
(5.8950)\end{array}$ \\
\hline 11 & PPC $(-1)$ & $\begin{array}{c}0.4894 \\
(1.2068)\end{array}$ & $\begin{array}{l}-3.0200^{* * *} \\
(-6.0611)\end{array}$ & $\begin{array}{l}-0.7198^{* * *} \\
(-2.8568)\end{array}$ & $\begin{array}{l}-1.8360^{* * *} \\
(-4.9070)\end{array}$ & $\begin{array}{l}0.0420 * * * \\
(5.4370)\end{array}$ & $\begin{array}{l}0.0276^{* * *} \\
(4.2350)\end{array}$ & $\begin{array}{c}0.0127^{*} \\
(1.6530)\end{array}$ & $\begin{array}{l}0.0183^{* * *} \\
(3.4450)\end{array}$ \\
\hline 12 & CLOAK $(-1)$ & $\begin{array}{r}-0.12281 \\
(-0.7380)\end{array}$ & $\begin{array}{c}0.3844^{*} \\
(1.8797)\end{array}$ & $\begin{array}{c}0.1176 \\
(1.1370)\end{array}$ & $\begin{array}{l}0.3329 * * \\
(2.1681)\end{array}$ & $\begin{array}{l}-0.0163 \\
(-1.0640)\end{array}$ & $\begin{array}{l}-0.0138 \\
(-1.0690)\end{array}$ & $\begin{array}{c}-0.0140 \\
(-0.9187)\end{array}$ & $\begin{array}{c}-0.0073 \\
(-0.6890)\end{array}$ \\
\hline 13 & BTS $(-1)$ & $\begin{array}{l}-0.6809^{* *} \\
(-2.1728)\end{array}$ & $\begin{array}{r}0.46371 \\
(1.2042)\end{array}$ & $\begin{array}{l}0.6770^{* * *} \\
(3.4764)\end{array}$ & $\begin{array}{l}1.3303^{* * *} \\
(4.6004)\end{array}$ & $\begin{array}{l}0.0327^{* * *} \\
(3.6690)\end{array}$ & $\begin{array}{l}0.0180^{* * *} \\
(2.3950)\end{array}$ & $\begin{array}{l}0.0274^{* * *} \\
(3.0940)\end{array}$ & $\begin{array}{c}-0.0103^{*} \\
(-1.6760)\end{array}$ \\
\hline 14 & $\operatorname{AEON}(-1)$ & $\begin{array}{l}-0.9784^{* * *} \\
(-4.1714)\end{array}$ & $\begin{array}{l}0.7939 * * * \\
(2.7546)\end{array}$ & $\begin{array}{c}0.1772 \\
(1.2160)\end{array}$ & $\begin{array}{l}1.0691 \text { *** } \\
(4.9397)\end{array}$ & $\begin{array}{l}0.0487^{* * * *} \\
(3.8170)\end{array}$ & $\begin{array}{l}0.0315^{* * *} \\
(2.9310)\end{array}$ & $\begin{array}{c}0.0123 \\
(0.9696)\end{array}$ & $\begin{array}{c}-0.0064 \\
(-0.7280)\end{array}$ \\
\hline 15 & XLM(-1) & $\begin{array}{c}0.0170 \\
(0.0783)\end{array}$ & $\begin{array}{c}0.4911^{*} \\
(1.8431)\end{array}$ & $\begin{array}{l}0.5639 * * * \\
(4.1814)\end{array}$ & $\begin{array}{l}0.5981^{* *} \\
(2.9892)\end{array}$ & $\begin{array}{l}-0.0031 \\
(-0.3247)\end{array}$ & $\begin{array}{l}-0.0268^{* * *} \\
(-3.3150)\end{array}$ & $\begin{array}{c}-0.0055 \\
(-0.3150)\end{array}$ & $\begin{array}{l}0.0145^{* *} \\
(2.1960)\end{array}$ \\
\hline 16 & $\operatorname{NAV}(-1)$ & $\begin{array}{l}-1.0730^{* * *} \\
(-5.0319)\end{array}$ & $\begin{array}{c}0.1297 \\
(0.4953)\end{array}$ & $\begin{array}{l}1.1108^{* * *} \\
(8.3859)\end{array}$ & $\begin{array}{l}1.2463^{* * *} \\
(6.3364)\end{array}$ & $\begin{array}{l}0.0613^{* * *} \\
(4.2940)\end{array}$ & $\begin{array}{l}0.0273^{* *} \\
(2.2660)\end{array}$ & $\begin{array}{l}-0.0288^{* *} \\
(-2.0240)\end{array}$ & $\begin{array}{c}0.0048 \\
(0.4846)\end{array}$ \\
\hline 17 & $\operatorname{NXT}(-1)$ & $\begin{array}{l}0.4986^{* *} \\
(2.4449)\end{array}$ & $\begin{array}{c}-0.0981 \\
(-0.3914)\end{array}$ & $\begin{array}{c}-0.0193 \\
(-0.1526)\end{array}$ & $\begin{array}{c}-0.2720 \\
(-1.4451)\end{array}$ & $\begin{array}{l}0.0087 \\
(0.9944)\end{array}$ & $\begin{array}{l}-0.0043 \\
(-0.5809)\end{array}$ & $\begin{array}{c}0.0006 \\
(0.0663)\end{array}$ & $\begin{array}{c}0.0076 \\
(1.2640)\end{array}$ \\
\hline 18 & $\mathrm{XVG}(-1)$ & $\begin{array}{c}-0.0340 \\
(-0.2168)\end{array}$ & $\begin{array}{l}-0.4594^{* *} \\
(-2.3863)\end{array}$ & $\begin{array}{c}0.0723 \\
(0.7427)\end{array}$ & $\begin{array}{c}0.0919 \\
(0.6356)\end{array}$ & $\begin{array}{l}0.1073^{* * *} \\
(6.4200)\end{array}$ & $\begin{array}{l}0.0538^{* * *} \\
(3.8140)\end{array}$ & $\begin{array}{l}0.0588^{* * *} \\
(3.5350)\end{array}$ & $\begin{array}{c}-0.0081 \\
(-0.7020)\end{array}$ \\
\hline 19 & MAID(-1) & $\begin{array}{c}-0.5737^{*} \\
(-1.7481)\end{array}$ & $\begin{array}{l}1.4236^{* * *} \\
(3.5305)\end{array}$ & $\begin{array}{c}-0.1705 \\
(-0.8360)\end{array}$ & $\begin{array}{c}-0.2948 \\
(-0.9736)\end{array}$ & $\begin{array}{l}0.0030 \\
(0.3909)\end{array}$ & $\begin{array}{l}-0.0061 \\
(-0.9620)\end{array}$ & $\begin{array}{c}-0.0144 * \\
(-1.9150)\end{array}$ & $\begin{array}{l}0.0121^{* *} \\
(2.3350)\end{array}$ \\
\hline 20 & XST(-1) & $\begin{array}{l}0.4933^{* * *} \\
(2.6938)\end{array}$ & $\begin{array}{l}-0.5072^{* *} \\
(-2.2542)\end{array}$ & $\begin{array}{l}-0.3578^{* * * *} \\
(-3.1445)\end{array}$ & $\begin{array}{c}-0.2360 \\
(-1.3968)\end{array}$ & $\begin{array}{l}0.0183 \\
(1.2540)\end{array}$ & $\begin{array}{l}0.0071 \\
(0.5770)\end{array}$ & $\begin{array}{c}0.0278^{*} \\
(1.9190)\end{array}$ & $\begin{array}{c}0.0007 \\
(0.0690)\end{array}$ \\
\hline
\end{tabular}

Note: This table reports the estimates for a fully specified Vector-Error-Correction Model using all twenty cryptocurrencies in our sample excluding Bitcoin. Our model uses daily data of $\log$ prices. The model has a lag-order of $p=2$. We report the estimates for the matrix $\boldsymbol{\beta}$ and the estimates for the adjustment parameter matrix $\boldsymbol{\alpha}$. The model allows for linear deterministic trend in data. The corresponding $t$-statistics are given in parentheses. The model is estimated with the econometric software Gretl. The sample period is from January 1, 2016 until December 31, 2018 corresponding to 1096 observations. Log-likelihood (lu) $=42519.662$.

*Statistically significant on a $10 \%$ level.

$* *$ Statistically significant on a $5 \%$ level.

$* * *$ Statistically significant on a $1 \%$ level.

We employ the Akaike criterion (AIC), Schwarz Bayesian criterion (BIC) and the Hannan-Quinn criterion (HQC), to assess the optimal lag-length od the Vector-Autoregression (VAR) model and find that the optimal lag-length is one for the HQC and BIC of two for the AIC (see Table A.1 in the appendix).

Next, using the optimal lag-length of two lags and running the trace test, we find that on the $5 \%$ level, there are four significant cointegration equilibrium relationships. The results are reported in Table $3 .{ }^{9}$

Since Bitcoin dominates the non-privacy cryptocurrency market, it would be useful to understand how the results are affected when adding Bitcoin to our sample. Adding Bitcoin to our set of cryptocurrencies and re-running the trace test, we find that including Bitcoin does not alter our main result (see Table 4); still there is evidence of four cointegration equilibria in the sample. Hence, our conclusions remain unchanged. While earlier studies found cointegration relationships in traditional currency markets (Norrbin, 1996; Woo, 1999; Haug et al., 2000; Rangvid and Sørensen, 2002; Aroskar et al., 2004) our novel findings suggest the presence of cointegration equilibria even in new digital currency markets.

\footnotetext{
${ }^{9}$ We decided to use two lags for the VECM specifications because testing for the optimal lag length in the VAR model representation suggests according to the AIC a lag-order of 2. The corresponding results are reported in Table A.1 in the appendix. Furthermore, the trend in data levels of non-privacy and privacy coins are reported in Fig. A.1 and Fig. A.2 in the appendix, which suggests to estimate the fully unrestricted VECM.
} 
Table 6

Restricted Vector-Error Correction model estimates using all 20 cryptocurrencies (excluding Bitcoin).

\begin{tabular}{|c|c|c|c|c|c|c|c|c|c|}
\hline$i$ & Coins & $\widehat{\boldsymbol{\beta}}_{1, i}$ & $\widehat{\boldsymbol{\beta}}_{2, i}$ & $\widehat{\boldsymbol{\beta}}_{3, i}$ & $\widehat{\boldsymbol{\beta}}_{4, i}$ & $\widehat{\boldsymbol{\alpha}}_{1, i}$ & $\widehat{\boldsymbol{\alpha}}_{2, i}$ & $\widehat{\alpha}_{3, i}$ & $\widehat{\boldsymbol{\alpha}}_{4, i}$ \\
\hline 1 & $\mathrm{XRP}(-1)$ & 4.9650 & 23.8620 & -4.4180 & 1.0840 & $\begin{array}{c}0.0020 * \\
(1.9370)\end{array}$ & $\begin{array}{c}0.0003 \\
(1.2750)\end{array}$ & $\begin{array}{l}0.0037^{* * *} \\
(3.6500)\end{array}$ & $\begin{array}{l}-0.0038 * * * \\
(-4.0660)\end{array}$ \\
\hline 2 & DASH(-1) & 1.4050 & 20.3200 & -5.4600 & 2.2000 & $\begin{array}{l}0.0030 * * * \\
(3.6390)\end{array}$ & $\begin{array}{c}-0.0002 \\
(-1.0270)\end{array}$ & $\begin{array}{c}0.0004 \\
(0.4767)\end{array}$ & $\begin{array}{l}0.0007 \\
(0.9418)\end{array}$ \\
\hline 3 & NMC(-1) & 1.0470 & -37.6080 & -6.7530 & -12.7680 & $\begin{array}{c}0.0026 \\
(1.9660)\end{array}$ & $\begin{array}{c}-0.0002 \\
(-0.6535)\end{array}$ & $\begin{array}{c}-0.0011 \\
(-0.8434)\end{array}$ & $\begin{array}{l}0.0033 \\
(2.8370)\end{array}$ \\
\hline 4 & $\operatorname{PXI}(-1)$ & 3.9350 & 8.8340 & 2.9110 & 5.0080 & $\begin{array}{l}-0.0065^{* *} \\
(-2.512)\end{array}$ & $\begin{array}{c}0.0013^{* *} \\
(2.5550)\end{array}$ & $\begin{array}{c}-0.0027 \\
(-1.0790)\end{array}$ & $\begin{array}{l}-0.0083^{* * *} \\
(-3.5770)\end{array}$ \\
\hline 5 & $\operatorname{LTC}(-1)$ & -6.1150 & 0.0000 & -1.8420 & -1.8950 & $\begin{array}{l}0.0036 * * * \\
(4.5920)\end{array}$ & $\begin{array}{c}-0.0002 \\
(-1.1500)\end{array}$ & $\begin{array}{c}0.0014^{*} \\
(1.8660)\end{array}$ & $\begin{array}{l}0.0004 \\
(0.5406)\end{array}$ \\
\hline 6 & $\mathrm{BCN}(-1)$ & -3.3150 & 31.2420 & 3.1460 & 6.5820 & $\begin{array}{l}0.0102^{* * *} \\
(6.6300)\end{array}$ & $\begin{array}{c}0.00002 \\
(0.0573)\end{array}$ & $\begin{array}{c}-0.0018 \\
(-1.1860)\end{array}$ & $\begin{array}{l}-0.0038^{* * *} \\
(-2.7810)\end{array}$ \\
\hline 7 & ETH(-1) & 3.3910 & 0.0000 & 3.8950 & 2.1490 & $\begin{array}{l}0.0021^{* *} \\
(2.3520)\end{array}$ & $\begin{array}{c}0.0001 \\
(0.3820)\end{array}$ & $\begin{array}{c}-0.0014 \\
(-1.6150)\end{array}$ & $\begin{array}{l}-0.0012 \\
(-1.2890)\end{array}$ \\
\hline 8 & $\mathrm{XMR}(-1)$ & -0.0490 & 66.3660 & 4.0290 & 4.8860 & $\begin{array}{l}0.0032^{* * *} \\
(3.2910)\end{array}$ & $\begin{array}{r}-0.00001 \\
(-0.0275)\end{array}$ & $\begin{array}{c}-0.0015 \\
(-1.6250)\end{array}$ & $\begin{array}{l}0.0009 \\
(0.9700)\end{array}$ \\
\hline 9 & DOGE $(-1)$ & 0.4290 & 0.0000 & 6.5460 & 6.3010 & $\begin{array}{l}0.0043 * * * \\
(4.4830)\end{array}$ & $\begin{array}{l}0.0007^{* * * *} \\
(3.4680)\end{array}$ & $\begin{array}{c}-0.0004 \\
(-0.4156)\end{array}$ & $\begin{array}{l}-0.0030 * * * \\
(-3.5040)\end{array}$ \\
\hline 10 & $\mathrm{XDN}(-1)$ & -7.3700 & -33.2890 & -9.5790 & -7.0180 & $\begin{array}{l}0.0107 * * * \\
(7.5970)\end{array}$ & $\begin{array}{l}0.0008^{* * * *} \\
(2.7990)\end{array}$ & $\begin{array}{c}0.0023^{*} \\
(1.6880)\end{array}$ & $\begin{array}{l}-0.0021 \\
(-1.6080)\end{array}$ \\
\hline 11 & $\operatorname{PPC}(-1)$ & -8.9510 & 0.0000 & 16.8170 & 0.8960 & $\begin{array}{l}0.0054 * * * \\
(5.6730)\end{array}$ & $\begin{array}{c}-0.0002 \\
(-0.6052)\end{array}$ & $\begin{array}{l}-0.0035^{* * *} \\
(-3.8050)\end{array}$ & $\begin{array}{l}0.0016 * \\
(1.8640)\end{array}$ \\
\hline 12 & CLOAK(-1) & 1.0890 & -9.6940 & -2.3870 & -1.5050 & $\begin{array}{c}-0.0032^{*} \\
(-1.7080)\end{array}$ & $\begin{array}{c}0.0004 \\
(1.1300)\end{array}$ & $\begin{array}{c}0.0021 \\
(1.1330)\end{array}$ & $\begin{array}{l}-0.0005 \\
(-0.2703)\end{array}$ \\
\hline 13 & BTS $(-1)$ & 3.6980 & 0.0000 & 1.3240 & 1.9320 & $\begin{array}{c}0.0014 \\
(1.2850)\end{array}$ & $\begin{array}{l}0.0009^{* * * *} \\
(3.9260)\end{array}$ & $\begin{array}{c}-0.0014 \\
(-1.3380)\end{array}$ & $\begin{array}{l}-0.0042^{* * *} \\
(-4.2180)\end{array}$ \\
\hline 14 & AEON(-1) & 0.2140 & -24.6440 & 0.3550 & 0.1280 & $\begin{array}{c}0.0007 \\
(0.4381)\end{array}$ & $\begin{array}{l}0.0012^{* * *} \\
(3.7950)\end{array}$ & $\begin{array}{l}-0.0034^{* *} \\
(-2.2330)\end{array}$ & $\begin{array}{l}-0.0021 \\
(-1.4560)\end{array}$ \\
\hline 15 & $\mathrm{XLM}(-1)$ & 3.8690 & 0.0000 & -4.4170 & -2.1640 & $\begin{array}{l}0.0027^{* *} \\
(2.3080)\end{array}$ & $\begin{array}{c}0.0002 \\
(0.8360)\end{array}$ & $\begin{array}{l}0.0049 * * * \\
(4.3380)\end{array}$ & $\begin{array}{l}-0.0028 * * * \\
(-2.6010)\end{array}$ \\
\hline 16 & NAV $(-1)$ & 1.1000 & -44.8930 & 0.8390 & -7.1840 & $\begin{array}{c}-0.0006 \\
(-0.3513)\end{array}$ & $\begin{array}{l}0.0018^{* * * *} \\
(4.9360)\end{array}$ & $\begin{array}{l}-0.0024 \\
(-1.4310)\end{array}$ & $\begin{array}{l}-0.0010 \\
(-0.6023)\end{array}$ \\
\hline 17 & NXT(-1) & 1.0430 & 0.0000 & -2.9420 & -2.2270 & $\begin{array}{c}0.0018^{*} \\
(1.6780)\end{array}$ & $\begin{array}{c}0.0002 \\
(1.0550)\end{array}$ & $\begin{array}{c}0.0013 \\
(1.2040)\end{array}$ & $\begin{array}{l}-0.0011 \\
(-1.0840)\end{array}$ \\
\hline 18 & $\operatorname{XVG}(-1)$ & -0.2140 & -3.2080 & 3.1300 & 0.1480 & $\begin{array}{l}0.0063 * * * \\
(3.0350)\end{array}$ & $\begin{array}{l}0.0026^{* * * *} \\
(6.0900)\end{array}$ & $\begin{array}{l}-0.0042^{* *} \\
(-2.1000)\end{array}$ & $\begin{array}{l}-0.0090 * * * \\
(-4.8370)\end{array}$ \\
\hline 19 & MAID(-1) & -2.5510 & 0.0000 & -6.4290 & -0.3290 & $\begin{array}{c}0.0012 \\
(1.2400)\end{array}$ & $\begin{array}{c}-0.0001 \\
(-0.4403)\end{array}$ & $\begin{array}{c}0.0010 \\
(1.1520)\end{array}$ & $\begin{array}{l}0.0009 \\
(1.0200)\end{array}$ \\
\hline 20 & $\mathrm{XST}(-1)$ & 0.5260 & 16.4420 & 2.5880 & 3.4220 & $\begin{array}{c}0.0032 * \\
(1.7580)\end{array}$ & $\begin{array}{c}0.0003 \\
(0.7125)\end{array}$ & $\begin{array}{c}-0.0001 \\
(-0.0739)\end{array}$ & $\begin{array}{l}-0.0026 \\
(-1.5830)\end{array}$ \\
\hline
\end{tabular}

Note: This table reports the estimates for a restricted Vector-Error-Correction Model using the set of all cryptocurrencies that are endogenous. Our model uses daily data of $\log$ prices. The model has a lag-order of $p=2$. We report the estimates for the matrix $\boldsymbol{\beta}$ and the estimates for the adjustment parameter matrix $\boldsymbol{\alpha}$. The model allows for linear deterministic trend in data. The corresponding $t$-statistics for the adjustment parameter matrix $\boldsymbol{\alpha}$ are given in parentheses. The model is estimated with the econometric software Gretl. The sample period is from January 1, 2016 until December 31 , 2018 corresponding to 1096 observations. Unrestricted loglikelihood $(\mathrm{lu})=42519.662$, Restricted loglikelihood $(\mathrm{lr})=42518.064,2 *(\mathrm{lu}-\mathrm{lr})=$ 3.19601, P(Chi-square(5) > 3.19601) $=0.669797$.

* Statistically significant on a $10 \%$ level.

** Statistically significant on a $5 \%$ level.

$* * *$ Statistically significant on a $1 \%$ level.

Establishing the existence of four cointegration relationships makes it possible to estimate the reduced form of model (3) using $\boldsymbol{\alpha} \boldsymbol{\beta}$ ' where the dimension of $\alpha$ and $\beta$ is 20x4. To test our hypothesis of interest, we order the vector $\boldsymbol{y}_{t-1}$ as,

$$
\begin{aligned}
& y_{t-1}=\left(X R P_{t-1}, D_{A S H} H_{t-1}, N M C_{t-1}, P X I_{t-1}, L T C_{t-1}, B C N_{t-1}, E T H_{t-1}, X M R_{t-1}, D O D G E_{t-1}, X D N_{t-1}, \cdots\right. \\
& \left.P P C_{t-1}, C L O A K_{t-1}, B T S_{t-1}, A E O N_{t-1}, X M L_{t-1}, N A V_{t-1}, N X T_{t-1}, X V G_{t-1}, M{ }_{t-1} D_{t-1}, X S T_{t-1}\right)
\end{aligned}
$$

The chosen ordering implies for the normalized matrix $\beta$ that,

$$
\boldsymbol{\beta}=\left[\boldsymbol{\beta}_{1}, \boldsymbol{\beta}_{2}, \boldsymbol{\beta}_{3}, \boldsymbol{\beta}_{4}\right]=\left[\begin{array}{cccc}
\beta_{X R P, 1} & 0 & 0 & 0 \\
0 & \beta_{D A S H, 2} & 0 & 0 \\
0 & 0 & \beta_{N M C, 3} & 0 \\
0 & 0 & 0 & \beta_{P X I, 4} \\
\beta_{L T C, 1} & \beta_{L T C, 2} & \beta_{L T C, 3} & \beta_{L T C, 4} \\
\vdots & \vdots & \vdots & \vdots \\
\beta_{X S T, 1} & \beta_{X S T, 2} & \beta_{X S T, 3} & \beta_{X S T, 4}
\end{array}\right]
$$




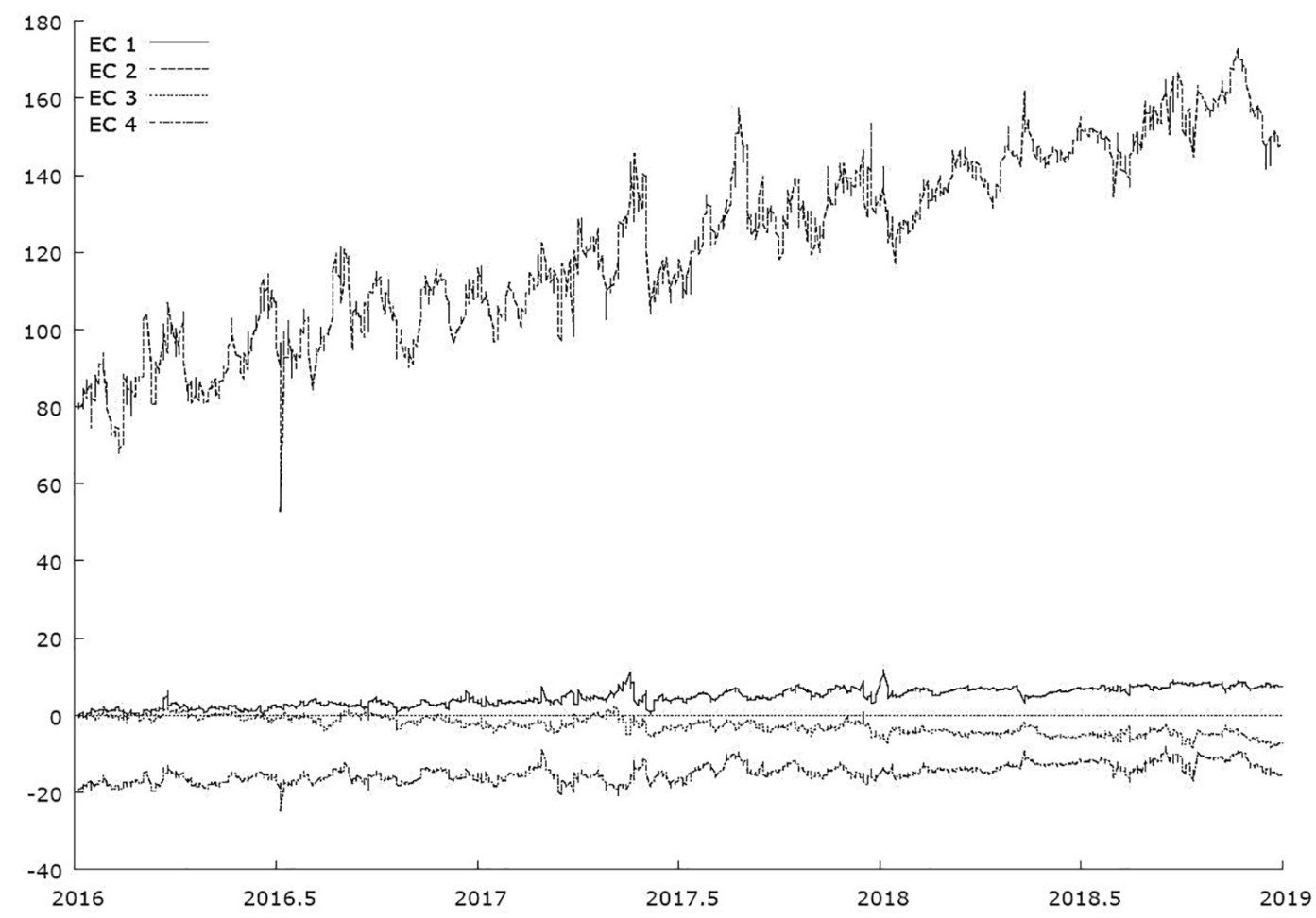

Fig. 1. This figure shows the cointegration equilibrium for privacy coins. The sample period is from January 1, 2016 until December 31,2018 corresponding to 1096 observations.

where $\boldsymbol{\beta}_{k}$ with $k=\{1, \cdots, 4\}$ are parameter vectors that have the dimension 20x1. Specifically, in our ordering we selected those cryptocurrencies that have the highest and lowest market capitalization in both categories, privacy coins (i.e., DASH and PXI) and nonprivacy coins (i.e., XRP and NMC) as the ones to be explained by the remaining cryptocurrencies. This approach allows us to identify whether the liquidity of cryptocurrencies matters for the formation of submarkets. Next, using our normalized matrix $\boldsymbol{\beta}$, we estimated a fully specified VECM using again all twenty cryptocurrencies. Since we are interested in the cointegration equilibria, we report only the point estimates for the matrices $\boldsymbol{\alpha}$ and $\boldsymbol{\beta}$, respectively. The results are reported in Table 5 .

Table 5 reveals some interesting results. First, the privacy coin market defined by PXI, which is in terms of market capitalization the smallest cryptocurrency investigated here, and defined by,

$$
\boldsymbol{\beta}_{4}{ }^{\prime}\left(\boldsymbol{y}_{t-1}-\mu_{0,4}-\mu_{1,4}(t-1)\right)
$$

seven out of eight non-privacy coins entering the cointegration equilibrium exhibit $t$-statistics indicating statistical significance on at least a 5\% level. This result implies that the submarket defined here is not a submarket detached from the market for non-privacy coins. Second, the privacy coin market defined by DASH, which is in terms of its market capitalization the largest cryptocurrency investigated here, and defined by,

$$
\boldsymbol{\beta}_{2}^{\prime}\left(\boldsymbol{y}_{t-1}-\mu_{0,2}-\mu_{1,2}(t-1)\right)
$$

only two out of eight non-privacy coins entering the cointegration equilibrium exhibit $t$-statistics indicating statistical significance on at least a $5 \%$ level. This result could indicate that this submarket defined is potentially detached from the market for non-privacy coins.

To test whether the submarket of privacy coins, defined by $\boldsymbol{\beta}_{2}{ }^{\prime}\left(\boldsymbol{y}_{t-1}-\mu_{0,2}-\mu_{1,2}(t-1)\right)$ is detached from the market for non-privacy coins, we set the following restrictions: $\beta_{2,5}=0, \beta_{2,7}=0, \beta_{2,9}=0, \beta_{2,11}=0, \beta_{2,13}=0, \beta_{2,15}=0, \beta_{2,17}=0, \beta_{2,19}=0$ and then reestimate the VECM. Note that the imposed restrictions imply that all eight non-privacy coins are set equal to zero and the equilibrium is formed by privacy coins only. Note also that our normalization accounts for $\beta_{2,1}=0$ and $\beta_{2,3}=0$ (i.e., $\beta_{2, X R P}=0$ and $\beta_{2, N M C}=$ 0 ) in the original model specification. The point estimates for $\boldsymbol{\alpha}$ and $\boldsymbol{\beta}$ for the restricted model are reported in Table 6.

The log-likelihood of the unrestricted model (Table 5) is 42519.66, whereas the log-likelihood of the unrestricted model is 42518.06. Employing the Likelihood-Ratio (LR) test gives us an estimated value of,

$$
\widehat{\lambda}=2(42519.66-42518.06)=3.20<11.07=\chi_{0.95}^{2}
$$

Since the estimated test statistic is clearly below the critical value of the corresponding reference distribution under null hypothesis 
Table 7

Vector-Error Correction model estimates using all 21 cryptocurrencies (including Bitcoin).

\begin{tabular}{|c|c|c|c|c|c|c|c|c|c|}
\hline$i$ & Coins & $\widehat{\boldsymbol{\beta}}_{1, i}$ & $\widehat{\boldsymbol{\beta}}_{2, i}$ & $\widehat{\boldsymbol{\beta}}_{3, i}$ & $\widehat{\boldsymbol{\beta}}_{4, i}$ & $\widehat{\boldsymbol{\alpha}}_{1, i}$ & $\widehat{\boldsymbol{\alpha}}_{2, i}$ & $\widehat{\boldsymbol{\alpha}}_{3, i}$ & $\widehat{\boldsymbol{\alpha}}_{4, i}$ \\
\hline 1 & $\mathrm{XRP}(-1)$ & $\begin{array}{c}1.0000 \\
(0.0000)\end{array}$ & $\begin{array}{c}0.0000 \\
(0.0000)\end{array}$ & $\begin{array}{c}0.0000 \\
(0.0000)\end{array}$ & $\begin{array}{c}0.0000 \\
(0.0000)\end{array}$ & $\begin{array}{l}-0.0076 \\
(-0.870)\end{array}$ & $\begin{array}{l}-0.0172 * * \\
(-2.1270)\end{array}$ & $\begin{array}{c}0.0004 \\
(0.0613)\end{array}$ & $\begin{array}{c}0.0076 \\
(1.4020)\end{array}$ \\
\hline 2 & DASH(-1) & $\begin{array}{c}0.0000 \\
(0.0000)\end{array}$ & $\begin{array}{c}1.0000 \\
(0.0000)\end{array}$ & $\begin{array}{c}0.0000 \\
(0.0000)\end{array}$ & $\begin{array}{c}0.0000 \\
(0.0000)\end{array}$ & $\begin{array}{l}0.0025 \\
(0.3580)\end{array}$ & $\begin{array}{l}-0.0036 \\
(-0.5500)\end{array}$ & $\begin{array}{l}-0.0008 \\
(-0.1675)\end{array}$ & $\begin{array}{l}0.0153^{* * *} \\
(3.5430)\end{array}$ \\
\hline 3 & NMC $(-1)$ & $\begin{array}{c}0.0000 \\
(0.0000)\end{array}$ & $\begin{array}{c}0.0000 \\
(0.0000)\end{array}$ & $\begin{array}{c}1.0000 \\
(0.0000)\end{array}$ & $\begin{array}{c}0.0000 \\
(0.0000)\end{array}$ & $\begin{array}{l}-0.0015 \\
(-0.1372)\end{array}$ & $\begin{array}{l}0.0091 \\
(0.8872)\end{array}$ & $\begin{array}{l}-0.0024 \\
(-0.3147)\end{array}$ & $\begin{array}{l}0.0146 \\
(2.1390) * *\end{array}$ \\
\hline 4 & $\operatorname{PXI}(-1)$ & $\begin{array}{c}0.0000 \\
(0.0000)\end{array}$ & $\begin{array}{c}0.0000 \\
(0.0000)\end{array}$ & $\begin{array}{c}0.0000 \\
(0.0000)\end{array}$ & $\begin{array}{c}1.0000 \\
(0.0000)\end{array}$ & $\begin{array}{l}0.0292 \\
(1.3410)\end{array}$ & $\begin{array}{l}0.0255 \\
(1.2660)\end{array}$ & $\begin{array}{l}0.0324^{* *} \\
(2.1740)\end{array}$ & $\begin{array}{l}-0.0524^{* * *} \\
(-3.8890)\end{array}$ \\
\hline 5 & $\operatorname{LTC}(-1)$ & $\begin{array}{l}-3.2199 * * * \\
(-5.4338)\end{array}$ & $\begin{array}{l}0.8418^{* * *} \\
(2.7834)\end{array}$ & $\begin{array}{l}3.1825^{* * *} \\
(3.1956)\end{array}$ & $\begin{array}{c}0.2241 \\
(0.6288)\end{array}$ & $\begin{array}{l}0.0090 \\
(1.3500)\end{array}$ & $\begin{array}{l}-0.0097 \\
(-1.5790)\end{array}$ & $\begin{array}{c}0.0011 \\
(0.2422)\end{array}$ & $\begin{array}{l}0.0170^{* * *} \\
(4.1440)\end{array}$ \\
\hline 6 & $\mathrm{BCN}(-1)$ & $\begin{array}{c}-0.1283 \\
(-0.3555)\end{array}$ & $\begin{array}{c}-0.0653 \\
(-0.3546)\end{array}$ & $\begin{array}{l}-0.3487 \\
(-0.5747)\end{array}$ & $\begin{array}{l}-0.6140^{* * *} \\
(-2.8271)\end{array}$ & $\begin{array}{l}0.0715^{* * *} \\
(5.5220)\end{array}$ & $\begin{array}{l}0.0272^{* *} \\
(2.2730)\end{array}$ & $\begin{array}{l}0.0429 * * * \\
(4.8310)\end{array}$ & $\begin{array}{l}0.0184^{* *} \\
(2.3010)\end{array}$ \\
\hline 7 & ETH $(-1)$ & $\begin{array}{c}0.4228 \\
(1.2135)\end{array}$ & $\begin{array}{l}-0.5460 * * * \\
(-3.0696)\end{array}$ & $\begin{array}{l}-0.1801 \\
(-0.3076)\end{array}$ & $\begin{array}{l}0.7983^{* * *} \\
(3.1123)\end{array}$ & $\begin{array}{l}0.0119^{*} \\
(1.6120)\end{array}$ & $\begin{array}{l}0.0150 * * \\
(2.1920)\end{array}$ & $\begin{array}{l}0.0108^{* *} \\
(2.1300)\end{array}$ & $\begin{array}{c}0.0021 \\
(0.4621)\end{array}$ \\
\hline 8 & $\mathrm{XMR}(-1)$ & $\begin{array}{l}-0.9931^{* *} \\
(-2.0396)\end{array}$ & $\begin{array}{l}-1.1750^{* * *} \\
(-4.7280)\end{array}$ & $\begin{array}{l}3.0670 * * * \\
(3.7483)\end{array}$ & $\begin{array}{l}-0.6145^{* *} \\
(-2.0980)\end{array}$ & $\begin{array}{l}0.0053 \\
(0.6446)\end{array}$ & $\begin{array}{l}0.0143^{*} \\
(1.8750)\end{array}$ & $\begin{array}{c}0.0064 \\
(1.1320)\end{array}$ & $\begin{array}{c}0.0096^{*} \\
(1.8870)\end{array}$ \\
\hline 9 & $\operatorname{DOGE}(-1)$ & $\begin{array}{l}-1.4630^{* * *} \\
(-3.4138)\end{array}$ & $\begin{array}{l}-0.02607 \\
(-0.1192)\end{array}$ & $\begin{array}{c}1.0270 \\
(1.4259)\end{array}$ & $\begin{array}{l}1.2550^{* * *} \\
(4.8685)\end{array}$ & $\begin{array}{l}0.0385^{* * *} \\
(4.7920)\end{array}$ & $\begin{array}{l}0.0114 \\
(1.5390)\end{array}$ & $\begin{array}{l}0.0256^{* * *} \\
(4.6610)\end{array}$ & $\begin{array}{c}0.0069 \\
(1.3840)\end{array}$ \\
\hline 10 & $\mathrm{XDN}(-1)$ & $\begin{array}{l}1.3271^{* * *} \\
(3.9482)\end{array}$ & $\begin{array}{l}0.9191^{* * *} \\
(5.3576)\end{array}$ & $\begin{array}{l}-2.8790^{* * *} \\
(-5.0964)\end{array}$ & $\begin{array}{l}-2.0910^{* * *} \\
(-10.3413)\end{array}$ & $\begin{array}{l}0.0533^{* * *} \\
(4.4480)\end{array}$ & $\begin{array}{l}-0.0002 \\
(-0.0224)\end{array}$ & $\begin{array}{l}0.0301^{* * *} \\
(3.6660)\end{array}$ & $\begin{array}{l}0.0383^{* * *} \\
(5.1720)\end{array}$ \\
\hline 11 & $\operatorname{PPC}(-1)$ & $\begin{array}{c}0.0423 \\
(0.0738)\end{array}$ & $\begin{array}{l}-2.5830 * * * \\
(-8.8348)\end{array}$ & $\begin{array}{c}-0.2824 \\
(-0.2934)\end{array}$ & $\begin{array}{l}-1.7190^{* * *} \\
(-4.9881)\end{array}$ & $\begin{array}{l}0.0303^{* * *} \\
(3.7830)\end{array}$ & $\begin{array}{l}0.0309^{* * *} \\
(4.1750)\end{array}$ & $\begin{array}{l}0.0176^{* * *} \\
(3.2110)\end{array}$ & $\begin{array}{l}0.0165^{* * *} \\
(3.3290)\end{array}$ \\
\hline 12 & $\operatorname{CLOAK}(-1)$ & $\begin{array}{l}-1.2639 * * * \\
(-4.8640)\end{array}$ & $\begin{array}{l}0.4614^{* * * *} \\
(3.4794)\end{array}$ & $\begin{array}{l}1.5613^{* * *} \\
(3.5751)\end{array}$ & $\begin{array}{l}0.8283^{* * *} \\
(5.2993)\end{array}$ & $\begin{array}{l}0.0061 \\
(0.3853)\end{array}$ & $\begin{array}{l}-0.0192 \\
(-1.3120)\end{array}$ & $\begin{array}{l}-0.0019 \\
(-0.1792)\end{array}$ & $\begin{array}{l}-0.0086 \\
(-0.8799)\end{array}$ \\
\hline 13 & BTS $(-1)$ & $\begin{array}{l}2.0520 * * * \\
(4.2823)\end{array}$ & $\begin{array}{c}-0.1234 \\
(-0.5041)\end{array}$ & $\begin{array}{l}-2.5420 * * * \\
(-3.1560)\end{array}$ & $\begin{array}{c}0.0164 \\
(0.0568)\end{array}$ & $\begin{array}{l}0.0281 * * * \\
(3.0490)\end{array}$ & $\begin{array}{l}0.0179 * * \\
(2.1030)\end{array}$ & $\begin{array}{l}0.0255^{* * *} \\
(4.0430)\end{array}$ & $\begin{array}{c}-0.0083 \\
(-1.4530)\end{array}$ \\
\hline 14 & $\operatorname{AEON}(-1)$ & $\begin{array}{c}-0.6005^{*} \\
(-1.7821)\end{array}$ & $\begin{array}{l}0.4365^{* * *} \\
(2.5380)\end{array}$ & $\begin{array}{l}-0.10107 \\
(-0.1785)\end{array}$ & $\begin{array}{l}0.8054 * * * \\
(3.9733)\end{array}$ & $\begin{array}{l}0.0378^{* * * *} \\
(2.8540)\end{array}$ & $\begin{array}{l}0.0318^{* * *} \\
(2.5940)\end{array}$ & $\begin{array}{l}0.0317 * * * \\
(3.4890)\end{array}$ & $\begin{array}{l}-0.0139^{*} \\
(-1.690)\end{array}$ \\
\hline 15 & XLM(-1) & $\begin{array}{l}-1.0560^{* * *} \\
(-3.3453)\end{array}$ & $\begin{array}{l}0.7228^{* * *} \\
(4.4880)\end{array}$ & $\begin{array}{l}1.8220 * * * \\
(3.4360)\end{array}$ & $\begin{array}{l}1.1150^{* * *} \\
(5.8756)\end{array}$ & $\begin{array}{l}-0.0086 \\
(-0.8655)\end{array}$ & $\begin{array}{l}-0.0339 * * * \\
(-3.6870)\end{array}$ & $\begin{array}{c}-0.0047 \\
(-0.6872)\end{array}$ & $\begin{array}{l}0.0142^{* *} \\
(2.3100)\end{array}$ \\
\hline 16 & $\operatorname{NAV}(-1)$ & $\begin{array}{l}-0.7261^{* * *} \\
(-5.3674)\end{array}$ & $\begin{array}{l}0.0948^{* *} \\
(2.0063)\end{array}$ & $\begin{array}{l}1.5061^{* * *} \\
(4.2492)\end{array}$ & $\begin{array}{c}0.2218 \\
(0.0271)\end{array}$ & $\begin{array}{l}-0.0013 \\
(-0.0883)\end{array}$ & $\begin{array}{l}0.0247^{*} \\
(1.7890)\end{array}$ & $\begin{array}{l}0.0212^{* *} \\
(2.0720)\end{array}$ & $\begin{array}{l}-0.0204 * * \\
(-2.2070)\end{array}$ \\
\hline 17 & NXT $(-1)$ & $\begin{array}{l}-0.7261^{* *} \\
(-2.3679)\end{array}$ & $\begin{array}{c}0.0948 \\
(0.6059)\end{array}$ & $\begin{array}{l}1.5061^{* * *} \\
(2.9224)\end{array}$ & $\begin{array}{c}0.2218 \\
(1.2024)\end{array}$ & $\begin{array}{l}0.0029 \\
(0.3190)\end{array}$ & $\begin{array}{l}-0.0086 \\
(-1.0270)\end{array}$ & $\begin{array}{c}0.0024 \\
(0.3853)\end{array}$ & $\begin{array}{c}0.0073 \\
(1.2960)\end{array}$ \\
\hline 18 & $\mathrm{XVG}(-1)$ & $\begin{array}{l}1.0755^{* * *} \\
(4.7743)\end{array}$ & $\begin{array}{l}-0.4860^{* * *} \\
(-4.2275)\end{array}$ & $\begin{array}{l}-1.3560^{* * *} \\
(-3.5814)\end{array}$ & $\begin{array}{l}-0.3850 * * * \\
(-2.8405)\end{array}$ & $\begin{array}{l}0.0789 * * * \\
(4.5510)\end{array}$ & $\begin{array}{l}0.0526 * * * \\
(3.2800)\end{array}$ & $\begin{array}{l}0.0706^{* * *} \\
(5.9410)\end{array}$ & $\begin{array}{c}-0.0123 \\
(-1.1450)\end{array}$ \\
\hline 19 & MAID(-1) & $\begin{array}{l}-1.1073^{* *} \\
(-2.3552)\end{array}$ & $\begin{array}{l}1.2521^{* * *} \\
(5.2182)\end{array}$ & $\begin{array}{c}0.57559 \\
(0.7284)\end{array}$ & $\begin{array}{l}-0.04574 \\
(-0.1617)\end{array}$ & $\begin{array}{l}-0.0048 \\
(-0.6112)\end{array}$ & $\begin{array}{l}-0.0093 \\
(-1.2850)\end{array}$ & $\begin{array}{c}-0.0051 \\
(-0.9578)\end{array}$ & $\begin{array}{l}0.0099 * * \\
(2.0560)\end{array}$ \\
\hline 20 & XST(-1) & $\begin{array}{l}-2.0344 * * * \\
(-7.4054)\end{array}$ & $\begin{array}{c}0.0583 \\
(0.4159)\end{array}$ & $\begin{array}{l}2.6091 * * * \\
(5.6501)\end{array}$ & $\begin{array}{l}0.9837^{* * *} \\
(5.9526)\end{array}$ & $\begin{array}{l}0.0508^{* * *} \\
(3.3790)\end{array}$ & $\begin{array}{l}0.0029 \\
(0.2049)\end{array}$ & $\begin{array}{l}0.0246^{* *} \\
(2.3910)\end{array}$ & $\begin{array}{c}0.0041 \\
(0.4403)\end{array}$ \\
\hline 21 & BTC $(-1)$ & $\begin{array}{l}4.3059 * * * \\
(6.3630)\end{array}$ & $\begin{array}{l}-1.1047^{* * *} \\
(-3.1986)\end{array}$ & $\begin{array}{l}-5.0060^{* * *} \\
(-4.4017)\end{array}$ & $\begin{array}{l}-2.0850^{* * *} \\
(-5.1220)\end{array}$ & $\begin{array}{l}0.0056 \\
(1.2340)\end{array}$ & $\begin{array}{l}0.0050 \\
(1.1780)\end{array}$ & $\begin{array}{c}0.0030 \\
(0.9537)\end{array}$ & $\begin{array}{c}0.0127 \\
(4.4910)\end{array}$ \\
\hline
\end{tabular}

Note: This table reports the estimates for a fully specified Vector-Error-Correction Model using all twenty-one cryptocurrencies in our sample including Bitcoin. Our model uses daily data of $\log$ prices. The model has a lag-order of $p=2$. We report the estimates for the matrix $\boldsymbol{\beta}$ and the estimates for the adjustment parameter matrix $\boldsymbol{\alpha}$. The model allows for linear deterministic trend in data. The corresponding $t$-statistics are given in parentheses. The model is estimated with the econometric software Gretl. The sample period is from January 1, 2016 until December 31, 2018 corresponding to 1096 observations. Log-likelihood(lu) $=45873.105$

*Statistically significant on a $10 \%$ level.

$* *$ Statistically significant on a $5 \%$ level.

$* * *$ Statistically significant on a $1 \%$ level.

which is chi-square distributed with five degrees of freedom, we cannot reject the null hypothesis ( $p$-value 0.6698). This result implies that the privacy coin market, as defined by $\boldsymbol{\beta}_{2}{ }^{\prime}\left(\boldsymbol{y}_{t-1}-\mu_{0,2}-\mu_{1,2}(t-1)\right)$ fulfills the conditions of a statistical equilibrium which is indeed detached from the market for non-privacy coins. In Fig. 1 we plot the cointegration equilibria $\boldsymbol{\beta}_{k}{ }^{\prime}\left(\boldsymbol{y}_{t-1}-\mu_{0}-\mu_{1}(t-1)\right)$ for $=\{1, \cdots, 4\}$, where $k=2$ defines our cointegration equilibrium for privacy coins. We observe from Fig. 1 that the cointegration equilibrium for privacy coins shows a clear linear stationary trend which underpins the necessity to employ a fully specified VECM accounting for both constant and trend term (see Equation (3)).

Next, we have shown that adding Bitcoin to our set of coins and re-running the trace test provided evidence that the system of equations still generated four cointegration equilibria. To test the robustness of our results, we again add Bitcoin to our sample so that $y_{t-1}$ is now a $21 \mathrm{x} 1$ vector given by

$$
\begin{aligned}
& y_{t-1}=\left(X R P_{t-1}, D A S H_{t-1}, N M C_{t-1}, P X I_{t-1}, L T C_{t-1}, B C N_{t-1}, E T H_{t-1}, X M R_{t-1}, D O D G E_{t-1}, X D N_{t-1}, \cdots\right. \\
& \left.P P C_{t-1}, C L O A K_{t-1}, B T S_{t-1}, A E O N_{t-1}, X M L_{t-1}, N A V_{t-1}, N X T_{t-1}, X V G_{t-1}, M A I D_{t-1}, X S T_{t-1}, B T C_{t-1}\right)
\end{aligned}
$$

Since we are interested in the cointegration equilibria, we again report only the point estimates for the matrices $\boldsymbol{\alpha}$ and $\boldsymbol{\beta}$, 
Table 8

Restricted Vector-Error Correction model estimates using all 21 cryptocurrencies (including Bitcoin).

\begin{tabular}{|c|c|c|c|c|c|c|c|c|c|}
\hline$i$ & Coins & $\widehat{\boldsymbol{\beta}}_{1, i}$ & $\widehat{\boldsymbol{\beta}}_{2, i}$ & $\widehat{\boldsymbol{\beta}}_{3, i}$ & $\widehat{\boldsymbol{\beta}}_{4, i}$ & $\widehat{\boldsymbol{\alpha}}_{1, i}$ & $\widehat{\boldsymbol{\alpha}}_{2, i}$ & $\widehat{\boldsymbol{\alpha}}_{3, i}$ & $\widehat{\boldsymbol{\alpha}}_{4, i}$ \\
\hline 1 & $\mathrm{XRP}(-1)$ & 2.7920 & -23.7910 & 0.2950 & 3.6640 & $\begin{array}{l}0.0031 * * * \\
(2.9240)\end{array}$ & $\begin{array}{l}-0.0001 \\
(-0.3261)\end{array}$ & $\begin{array}{c}0.0010 \\
(0.9609)\end{array}$ & $\begin{array}{c}0.0012 \\
(1.2960)\end{array}$ \\
\hline 2 & DASH $(-1)$ & 0.6610 & -19.1560 & -5.4660 & 0.2790 & $\begin{array}{l}0.0033^{* * *} \\
(4.0070)\end{array}$ & $\begin{array}{l}0.0001 \\
(0.8537)\end{array}$ & $\begin{array}{c}0.0003 \\
(0.3134)\end{array}$ & $\begin{array}{c}-0.0005 \\
(-0.6296)\end{array}$ \\
\hline 3 & NMC(-1) & 0.1800 & 31.0960 & -3.9900 & 12.3180 & $\begin{array}{l}0.0029^{* * *} \\
(2.186)\end{array}$ & $\begin{array}{l}0.00003 \\
(0.1268)\end{array}$ & $\begin{array}{c}-0.0018 \\
(-1.3880)\end{array}$ & $\begin{array}{l}-0.0023^{* *} \\
(-2.025)\end{array}$ \\
\hline 4 & $\operatorname{PXI}(-1)$ & 4.3980 & -7.8020 & 2.3050 & -3.9460 & $\begin{array}{l}-0.0076^{* * *} \\
(-3.0060)\end{array}$ & $\begin{array}{l}-0.0011^{* *} \\
(-2.1690)\end{array}$ & $\begin{array}{c}-0.0012 \\
(-0.4614)\end{array}$ & $\begin{array}{l}0.0071^{* * *} \\
(3.1430)\end{array}$ \\
\hline 5 & $\operatorname{LTC}(-1)$ & -3.6900 & 0.0000 & -8.8260 & -4.0540 & $\begin{array}{l}0.0034 * * * \\
(4.2730)\end{array}$ & $\begin{array}{l}0.0001 \\
(0.4629)\end{array}$ & $\begin{array}{c}0.0018^{* *} \\
(2.3360)\end{array}$ & $\begin{array}{c}0.0002 \\
(0.3161)\end{array}$ \\
\hline 6 & $\mathrm{BCN}(-1)$ & -2.7300 & -26.8060 & 1.7350 & -7.4520 & $\begin{array}{l}0.0079 * * * \\
(5.1460)\end{array}$ & $\begin{array}{l}-0.0005 \\
(-1.5720)\end{array}$ & $\begin{array}{c}-0.0001 \\
(-0.0748)\end{array}$ & $\begin{array}{l}0.0070^{* * *} \\
(5.2810)\end{array}$ \\
\hline 7 & $\operatorname{ETH}(-1)$ & 3.4570 & 0.0000 & 4.7460 & -1.3560 & $\begin{array}{l}0.0022^{* *} \\
(2.4650)\end{array}$ & $\begin{array}{l}-0.0001 \\
(-0.4883)\end{array}$ & $\begin{array}{l}-0.0020 * * \\
(-2.3180)\end{array}$ & $\begin{array}{c}0.0012 \\
(1.6270)\end{array}$ \\
\hline 8 & XMR $(-1)$ & -1.4310 & -64.3710 & 4.3720 & -4.8660 & $\begin{array}{l}0.0034^{* * *} \\
(3.5030)\end{array}$ & $\begin{array}{l}-0.00008 \\
(-0.42620)\end{array}$ & $\begin{array}{l}-0.0026^{* * *} \\
(-2.6420)\end{array}$ & $\begin{array}{c}-0.0003 \\
(-0.3547)\end{array}$ \\
\hline 9 & DOGE(-1) & 2.6610 & 0.0000 & 1.7870 & -9.1560 & $\begin{array}{l}0.0035 * * * \\
(3.6700)\end{array}$ & $\begin{array}{l}-0.0008^{* * *} \\
(-4.0000)\end{array}$ & $\begin{array}{c}0.0006 \\
(0.5781)\end{array}$ & $\begin{array}{l}0.0036^{* * *} \\
(4.3010)\end{array}$ \\
\hline 10 & $\mathrm{XDN}(-1)$ & -8.5870 & 33.4290 & -6.7550 & 8.2280 & $\begin{array}{l}0.0099 * * * \\
(7.0250)\end{array}$ & $\begin{array}{l}-0.0011^{* * *} \\
(-3.8160)\end{array}$ & $\begin{array}{c}0.0028 * \\
(1.9610)\end{array}$ & $\begin{array}{l}0.0029^{* *} \\
(2.3340)\end{array}$ \\
\hline 11 & $\operatorname{PPC}(-1)$ & -8.0090 & 0.0000 & 14.2560 & -7.3220 & $\begin{array}{l}0.0051^{* * *} \\
(5.3590)\end{array}$ & $\begin{array}{l}-0.0001 \\
(-0.6383)\end{array}$ & $\begin{array}{l}-0.0032^{* * *} \\
(-3.3300)\end{array}$ & $\begin{array}{c}0.0006 \\
(0.7335)\end{array}$ \\
\hline 12 & CLOAK(-1) & 1.6940 & 10.7940 & -4.2270 & 1.0230 & $\begin{array}{l}-0.0042^{* *} \\
(-2.2340)\end{array}$ & $\begin{array}{l}-0.0005 \\
(-1.2590)\end{array}$ & $\begin{array}{l}0.0047 * * \\
(2.5280)\end{array}$ & $\begin{array}{c}0.0004 \\
(0.2280)\end{array}$ \\
\hline 13 & BTS $(-1)$ & 2.8940 & 0.0000 & 4.3040 & 1.1000 & $\begin{array}{c}0.0011 \\
(0.9834)\end{array}$ & $\begin{array}{l}-0.0008 * * * \\
(-3.7300)\end{array}$ & $\begin{array}{c}-0.0012 \\
(-1.1370)\end{array}$ & $\begin{array}{l}0.0037^{* * *} \\
(3.8850)\end{array}$ \\
\hline 14 & $\operatorname{AEON(-1)}$ & 1.5060 & 24.8650 & -1.8870 & -1.1170 & $\begin{array}{c}-0.0006 \\
(-0.3696)\end{array}$ & $\begin{array}{l}-0.0013^{* * *} \\
(-4.2610)\end{array}$ & $\begin{array}{c}-0.0021 \\
(-1.3250)\end{array}$ & $\begin{array}{l}0.0030 * * \\
(2.2220)\end{array}$ \\
\hline 15 & $\mathrm{XLM}(-1)$ & 3.9810 & 0.0000 & -5.6250 & 2.8620 & $\begin{array}{l}0.0032^{* * *} \\
(2.6700)\end{array}$ & $\begin{array}{l}-0.0001 \\
(-0.3685)\end{array}$ & $\begin{array}{l}0.0038^{* * *} \\
(3.2000)\end{array}$ & $\begin{array}{c}0.0007 \\
(0.6931)\end{array}$ \\
\hline 16 & $\mathrm{NAV}(-1)$ & 1.1950 & 39.9050 & 3.0150 & 6.6620 & $\begin{array}{c}-0.0010 \\
(-0.5785)\end{array}$ & $\begin{array}{l}-0.0018 * * * \\
(-5.1130)\end{array}$ & $\begin{array}{l}-0.0047^{* * *} \\
(-2.6730)\end{array}$ & $\begin{array}{c}0.00003 \\
(0.0217)\end{array}$ \\
\hline 17 & NXT(-1) & 0.4050 & 0.0000 & -2.7740 & 2.9580 & $\begin{array}{c}0.0019 * \\
(1.7750)\end{array}$ & $\begin{array}{l}-0.0002 \\
(-0.9222)\end{array}$ & $\begin{array}{c}0.0012 \\
(1.0740)\end{array}$ & $\begin{array}{c}0.0006 \\
(0.5848)\end{array}$ \\
\hline 18 & $\mathrm{XVG}(-1)$ & -0.4000 & 1.6220 & 4.2720 & -0.2050 & $\begin{array}{l}0.0049^{* *} \\
(2.3800)\end{array}$ & $\begin{array}{l}-0.0025^{* * *} \\
(-6.2330)\end{array}$ & $\begin{array}{c}-0.0039 * \\
(-1.9210)\end{array}$ & $\begin{array}{l}0.0087^{* * *} \\
(4.8620)\end{array}$ \\
\hline 19 & MAID(-1) & -1.9200 & 0.0000 & -8.4200 & -0.4170 & $\begin{array}{c}0.0013 \\
(1.6440)\end{array}$ & $\begin{array}{l}-0.0005 \\
(0.6050)\end{array}$ & $\begin{array}{c}0.0037 \\
(1.0090)\end{array}$ & $\begin{array}{c}0.0047 \\
(-1.2690)\end{array}$ \\
\hline 20 & $\mathrm{XST}(-1)$ & 1.8850 & -14.8190 & -1.4020 & -5.6850 & $\begin{array}{c}0.0013 \\
(0.7027)\end{array}$ & $\begin{array}{l}-0.0005 \\
(-1.4040)\end{array}$ & $\begin{array}{l}0.0037^{* *} \\
(2.1020)\end{array}$ & $\begin{array}{l}0.0047^{* * *} \\
(3.0230)\end{array}$ \\
\hline 21 & BTC $(-1)$ & -3.1250 & 0.0000 & 9.9740 & 5.4360 & $\begin{array}{l}0.0032 * * * \\
(5.9210)\end{array}$ & $\begin{array}{l}0.00001 \\
(0.0585)\end{array}$ & $\begin{array}{c}-0.0009 \\
(-1.5900)\end{array}$ & $\begin{array}{c}-0.0004 \\
(-0.8382)\end{array}$ \\
\hline
\end{tabular}

Note: This table reports the estimates for a restricted Vector-Error-Correction Model using the set of all cryptocurrencies that are endogenous including Bitcoin. Our model uses daily data of $\log$ prices. The model has a lag-order of $p=2$. We report the estimates for the matrix $\boldsymbol{\beta}$ and the estimates for the adjustment parameter matrix $\boldsymbol{\alpha}$. The model allows for linear deterministic trend in data. The corresponding t-statistics for the adjustment parameter matrix $\boldsymbol{\alpha}$ are given in parentheses. The model is estimated with the econometric software Gretl. The sample period is from January 1, 2016 until December 31, 2018 corresponding to 1096 observations. Unrestricted loglikelihood (lu) = 45873.105, Restricted loglikelihood $(\mathrm{lr})=45869.056,2 *(\mathrm{lu}-\mathrm{lr})=8.09739, \mathrm{P}($ Chi-square $(6)>8.09739)=0.231055$

* Statistically significant on a $10 \%$ level.

** Statistically significant on a $5 \%$ level.

$* * *$ Statistically significant on a $1 \%$ level.

respectively. The results are reported in Table 7.

Again, we normalized the matrix $\beta$ which is now of dimension $21 \times 4$, and the choose the same ordering as before, that is,

$$
\boldsymbol{\beta}=\left[\boldsymbol{\beta}_{1}, \boldsymbol{\beta}_{2}, \boldsymbol{\beta}_{3}, \boldsymbol{\beta}_{4}\right]=\left[\begin{array}{ccccc}
\beta_{X R P, 1} & 0 & & 0 & 0 \\
0 & \beta_{D A S H, 2} & & 0 & 0 \\
0 & 0 & \beta_{N M C, 3} & 0 \\
0 & 0 & 0 & \beta_{P X I, 4} \\
\beta_{L T C, 1} & \beta_{L T C, 2} & \beta_{L T C, 3} & \beta_{L T C, 4} \\
\vdots & \vdots & \vdots & \vdots \\
\beta_{X S T, 1} & \beta_{X S T, 2} & \beta_{X S T, 3} & \beta_{X S T, 4} \\
\beta_{B T C, 1} & \beta_{B T C, 2} & \beta_{B T C, 3} & \beta_{B T C, 4}
\end{array}\right]
$$

where $\boldsymbol{\beta}_{k}$ with $k=\{1, \cdots, 4\}$ are parameter vectors that have the dimension $21 \mathrm{x} 1$. Again, we test whether the submarket of privacy coins, defined by $\boldsymbol{\beta}_{2}{ }^{\prime}\left(\boldsymbol{y}_{t-1}-\mu_{0,2}-\mu_{1,2}(t-1)\right)$ is detached from the market for non-privacy coins by re-estimating the VECM using the 
following restrictions: $\beta_{2,5}=0, \beta_{2,7}=0, \beta_{2,9}=0, \beta_{2,11}=0, \beta_{2,13}=0, \beta_{2,15}=0, \beta_{2,17}=0, \beta_{2,19}=0$, and additionally $\beta_{2,21}=0$. The imposed restrictions imply that all nine non-privacy coins including Bitcoin are set equal to zero, which means that the equilibrium is formed by privacy coins only. Note again that our chosen normalization accounts for $\beta_{2,1}=0$ and $\beta_{2,3}=0$ (e.g., $\beta_{2, X R P}=0$ and $\beta_{2, N M C}=0$ ) in the original model specification. The point estimates for $\boldsymbol{\alpha}$ and $\boldsymbol{\beta}$ for the restricted model are reported in Table 8 .

The log-likelihood of the unrestricted model (Table 7) is 45873.11, whereas the log-likelihood of the unrestricted restricted model is 45869.06. Employing the Likelihood-Ratio (LR) test gives us an estimated value of

$$
\widehat{\lambda}=2(45873.11-45869.06)=8.10<12.59=\chi_{0.95}^{2}
$$

Since the estimated test statistic is below the critical value of the corresponding reference distribution under null hypothesis which is chi-square distributed with six degrees of freedom, we cannot reject the null hypothesis ( $p$-value 0.2311). This result implies that despite of accounting for Bitcoin as the cryptocurrency that exhibits the highest market capitalization the privacy coin market, as defined by $\boldsymbol{\beta}_{2}{ }^{\prime}\left(\boldsymbol{y}_{t-1}-\mu_{0,2}-\mu_{1,2}(t-1)\right)$ fulfills the conditions of a statistical equilibrium. This statistical equilibrium is detached from the market for non-privacy coins. Hence, the results of our robustness check strongly confirms our previous findings.

\section{Conclusion}

Goldfeder et al. (2018) show how third-party web trackers can de-anonymize users of Bitcoin and other non-privacy coins. Due to financial transparency, institutions are hesitant to use non-privacy cryptocurrencies as a medium of exchange. The emerge of privacy coins remedies this issue by using features like masternode technology, ring signature, and a stealth wallet address, to make it impossible for third parties to trace transactions to the real parties involved. We hypothesized that the traders, who favor privacy over complete transparency, are emerging as a different subgroup in digital financial markets. A common feature of cryptocurrencies is that the total supply of cryptocurrencies is often predetermined. As a consequence, the price processes depend solely on the demand side, that is, the users. Given that the user base for privacy coins is different from that for non-privacy coins, we would expect that privacy coins form a submarket of cryptocurrencies that is detached from the market for non-privacy coins.

To explore this issue, we make use of cointegration analysis which has at least three benefits; first, we can test whether the market for privacy coins generates a cointegration equilibrium that is detached from the market for non-privacy coins. Second, given that a cointegration equilibrium exists, the model allows us to test at the same for market efficiency. Third, and finally, making use of this model has the advantage that is does not require the specific formulation of an equilibrium price mechanism. Using the whole set of cryptocurrencies consisting of twenty cryptocurrencies to estimate the model, we find evidence for four cointegration equilibria. Accounting for liquidity, we estimate a fully-specified VECM by selecting the privacy and non-privacy coins with highest and lowest market capitalization as the four left-hand side variables in the model. Whereas in the equation modeling the privacy coin with lowest market capitalization (PXI) enter both categories of cryptocurrencies, that is, privacy and non-privacy coins, in the equation modeling DASH, which is the privacy coin with highest market capitalization, only two of non-privacy coins entering the equation exhibit statistical significance. Performing a Likelihood-Ratio test, we find that we cannot reject the null hypothesis that the whole set of nonprivacy coins is jointly insignificant.

First, an immediate implication of cointegration is the existence of Granger-causal orderings among cointegrated series, which implies that asset prices determined in a weakly efficient market cannot be cointegrated. Hence, our findings provide evidence for market inefficiency. Second, the cointegration equilibrium associated with DASH appears to be disconnected from the market for nonprivacy coins. A novel aspect of our study is it providing evidence that the underlying forces that cause large cap privacy coins equilibrium are unrelated to those at work in the non-privacy coins market. One explanation could be behavioral type: It could be that the market actors in the privacy coin market are different from those that trade in the non-privacy coin market. For instance, criminals involved in money laundry could favor privacy coins exhibiting a high level of liquidity as the sums involved could be substantial making small cap privacy coins an inappropriate choice for money laundry.

However, future studies might explore the market heterogeneity in the cryptocurrency market in more detail. Also, potential factors that might have caused the cointegration relationships should be the subject of future research. Third, if the stable cointegration relationship between asset prices is known to the market participants they would be able to exploit it and be in a position to profit. There is a broad stream of literature dealing with pairs trading, for instance, that requires the presence of cointegrated assets. Future research investigating this issue in the context of new digital currency markets would be welcome. Finally, since cryptocurrencies are by definition privy of intrinsic value - unlike fiat money issued by governments - the extent to which shocks may propagate across cryptocurrencies raises questions as to whether any observed spillover stems from investors' rebalancing activity and the accompanying price pressures rather than from fundamental information transmission. Future research is needed to elaborate on these mechanisms.

\section{Declaration of Competing Interest}

The authors declare that they have no known competing financial interests or personal relationships that could have appeared to influence the work reported in this paper. 


\section{Appendix A}

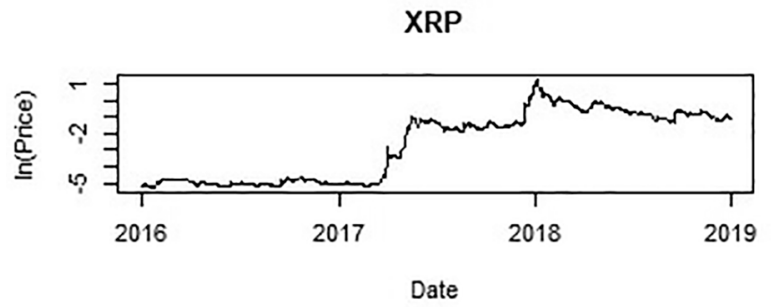

LTC

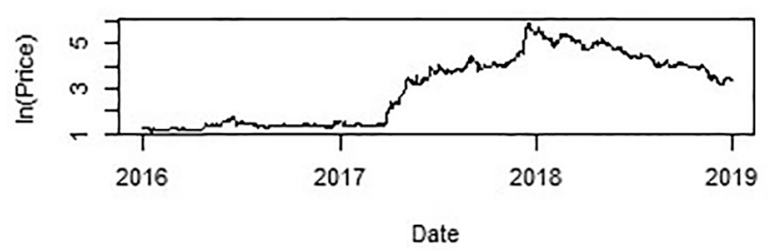

ETH

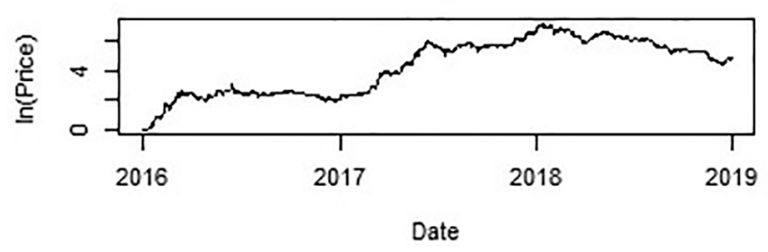

PPC

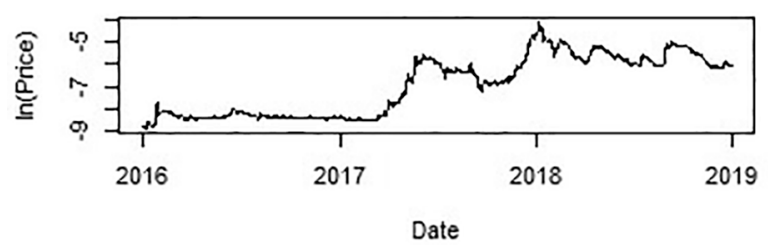

BTS

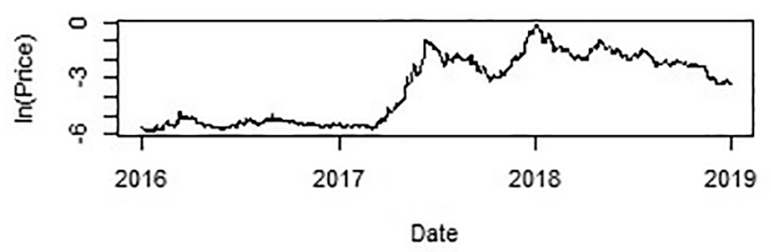

XLM

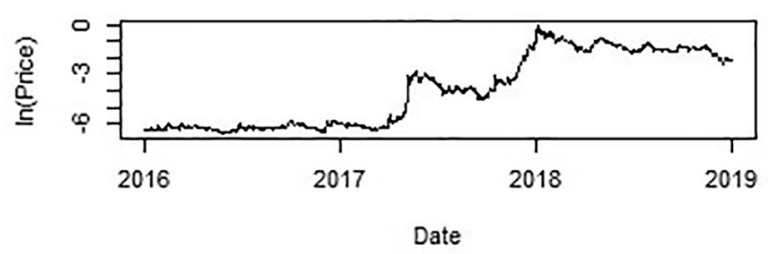

NXT

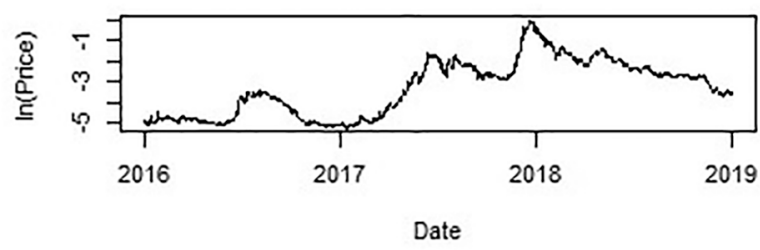

MAID

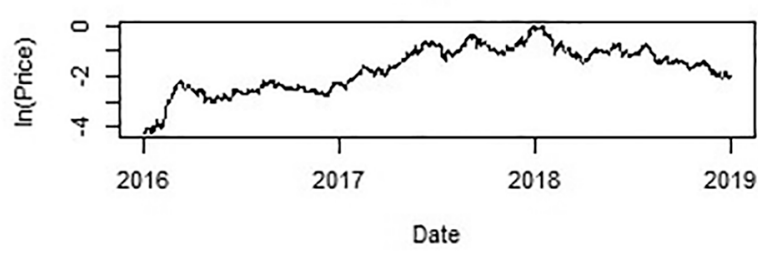

PPC

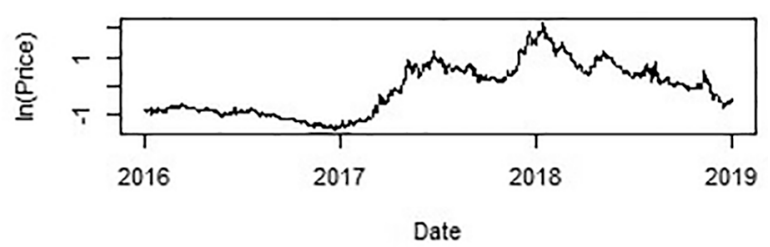

NMC

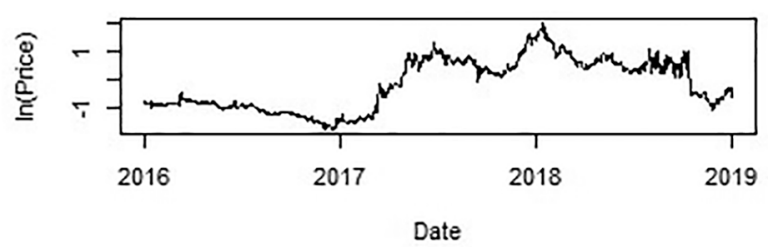

Fig. A1. Trend in the data levels of top 10 non-privacy coins 2016-2018(excluding Bitcoin). 
DASH

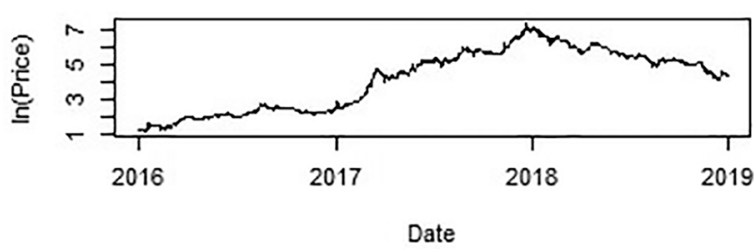

BCN

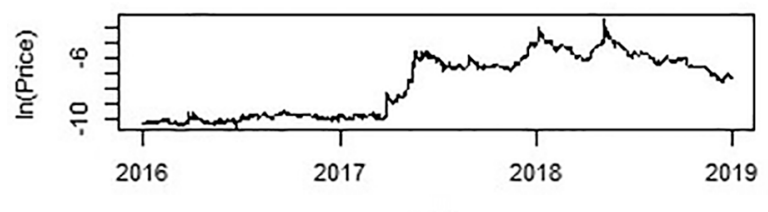

Date

XDN

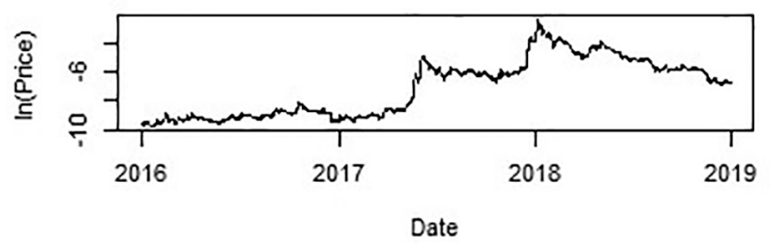

XMR

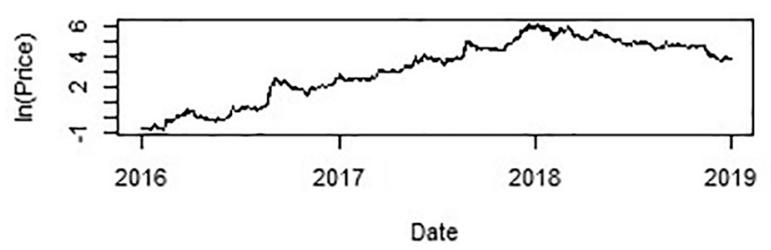

CLOAK

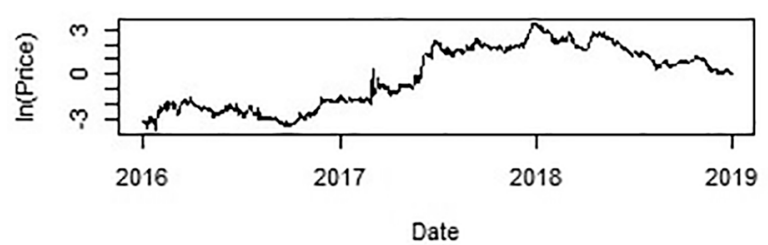

AEON

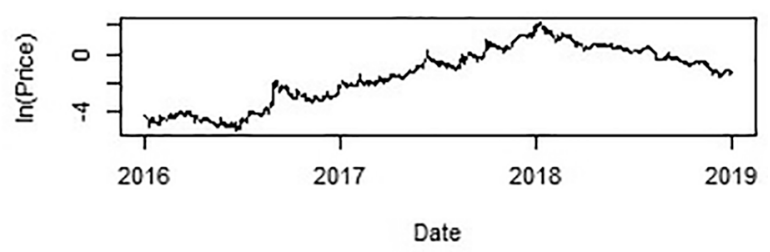

XST

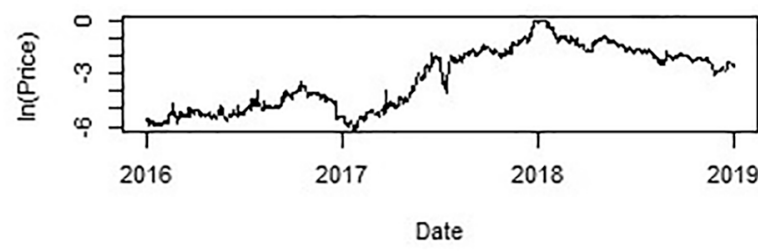

PXI

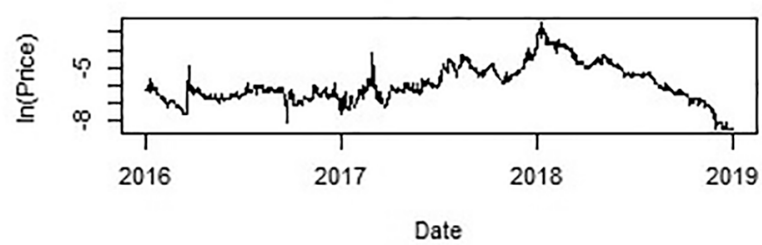

NAV

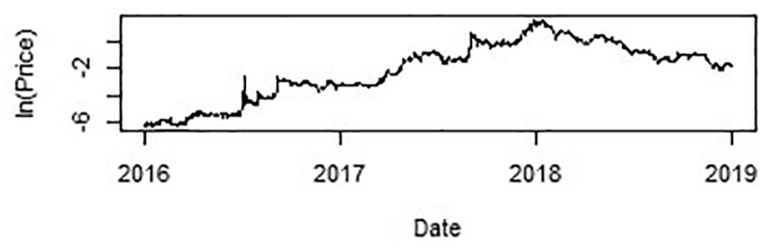

XVG

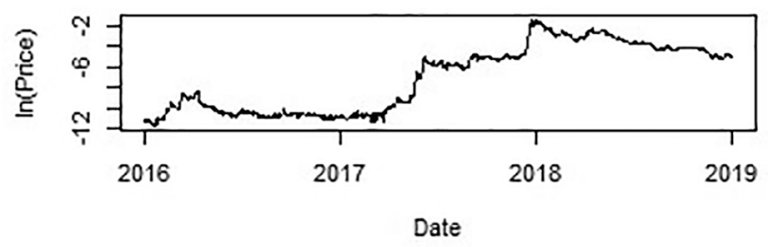

Fig. A2. Trend in the data levels of top 10 privacy coins 2016-2018. 
Table A1

VAR optimal lag order selection.

\begin{tabular}{|c|c|c|c|c|c|}
\hline lags & loglik & $\mathrm{p}(\mathrm{LR})$ & AIC & BIC & HQC \\
\hline 1 & 40392.0140 & & -74.3365 & $-72.4894 *$ & $-73.6369 *$ \\
\hline 2 & 40898.6327 & 0.0000 & $-74.6071 *$ & -71.0889 & -73.2747 \\
\hline 3 & 41194.6165 & 0.0000 & -74.4863 & -69.2970 & -72.5210 \\
\hline 4 & 41495.7699 & 0.0000 & -74.3750 & -67.5146 & -71.7769 \\
\hline 5 & 41753.5155 & 0.0000 & -74.1831 & -65.6515 & -70.9521 \\
\hline 6 & 42007.6063 & 0.0000 & -73.9844 & -63.7817 & -70.1205 \\
\hline 7 & 42302.2864 & 0.0000 & -73.8611 & -61.9873 & -69.3643 \\
\hline 8 & 42549.7210 & 0.0000 & -73.6500 & -60.1050 & -68.5203 \\
\hline 9 & 42845.2508 & 0.0000 & -73.5283 & -58.3122 & -67.7658 \\
\hline 10 & 43120.1391 & 0.0000 & -73.3683 & -56.4810 & -66.9728 \\
\hline 11 & 43442.7987 & 0.0000 & -73.2970 & -54.7386 & -66.2687 \\
\hline 12 & 43730.9191 & 0.0000 & -73.1616 & -52.9320 & -65.5003 \\
\hline 13 & 44012.0285 & 0.0000 & -73.0131 & -51.1124 & -64.7189 \\
\hline 14 & 44335.3340 & 0.0000 & -72.9430 & -49.3712 & -64.0160 \\
\hline 15 & 44645.5180 & 0.0000 & -72.8485 & -47.6056 & -63.2886 \\
\hline 16 & 44942.0211 & 0.0000 & -72.7287 & -45.8146 & -62.5359 \\
\hline 17 & 45257.0731 & 0.0000 & -72.6433 & -44.0580 & -61.8176 \\
\hline 18 & 45574.6329 & 0.0000 & -72.5625 & -42.3061 & -61.1039 \\
\hline 19 & 45894.2404 & 0.0000 & -72.4856 & -40.5581 & -60.3941 \\
\hline 20 & 46206.6556 & 0.0000 & -72.3953 & -38.7966 & -59.6709 \\
\hline
\end{tabular}

Note: VAR system, maximum lag order 20

The asterisks indicate the best (that is, minimized) values

of the respective information criteria, AIC = Akaike criterion,

$\mathrm{BIC}=$ Schwarz Bayesian criterion and HQC = Hannan-Quinn criterion.

\section{Appendix B. Supplementary material}

Supplementary data to this article can be found online at https://doi.org/10.1016/j.intfin.2021.101402.

\section{References}

Ahmed, S., Grobys, K., Sapkota, N., 2020. Profitability of Technical Trading Rules among Cryptocurrencies with Privacy Function. Finance Res. Lett. 35 , 101495. https://doi.org/10.1016/j.frl.2020.101495.

Alexander, C., Dimitriu, A., 2005. Indexing Cointegration and Equity Market Regimes. J. Int. Finance Econ. 10 (3), $213-231$.

Al-Yahyaee, K.H., Mensi, W., Yoon, S.-M., 2018. Efficiency, multifractality, and the long-memory property of the Bitcoin market: A comparative analysis with stock, currency, and gold markets. Finance Res. Lett. 27, 228-234.

Androulaki, E., Karame, G.O., Roeschlin, M., Scherer, T. and Capkun, S., 2013, April. Evaluating user privacy in bitcoin. In International Conference on Financial Cryptography and Data Security (pp. 34-51). Springer, Berlin, Heidelberg.

Aroskar, R., Sarkar, S.K., Swanson, P.E., 2004. European foreign exchange market efficiency: Evidence based on crisis and noncrisis periods. Int. Rev. Financial Analy. 13 (3), 333-347.

Bariviera, A.F., 2017. The inefficiency of Bitcoin revisited: A dynamic approach. Economics Letters 161, 1-4.

Baur, D.G., Hong, K., Lee, A.D., 2018. Bitcoin: Medium of exchange or speculative assets? J. Int. Financial Markets, Inst. Money 54, $177-189$.

Borri, N., 2019. Conditional tail-risk in cryptocurrency markets. J. Empirical Finance 50, 1-19.

Bouri, E., Molnár, P., Azzi, G., Roubaud, D., Hagfors, L.I., 2017. On the hedge and safe haven properties of Bitcoin: Is it really more than a diversifier? Finance Res. Lett. 20, 192-198.

Brenig, C., Accorsi, R., Müller, G., 2015, May. Economic Analysis of Cryptocurrency Backed Money Laundering. In ECIS.

Copeland, L.S., 1991. Cointegration tests with daily exchange rate data. Oxford Bull. Econ. Stat. 53 (2), $185-198$.

Corbet, S., Eraslan, V., Lucey, B., Sensoy, A., 2019. The effectiveness of technical trading rules in cryptocurrency markets. Finance Res. Lett. 31, 32-37.

Dyhrberg, A.H., 2016. Hedging capabilities of bitcoin. Is it the virtual gold? Finance Res. Lett. 16, 139-144.

Engle, R.F., Granger, C.W.J., 1987. Cointegration and error correction: representation, estimation, and testing. Econometrica 55 (2), 251. https://doi.org/10.2307/ 1913236.

Foley, S., Karlsen, J.R., Putninsš, T.J., 2019. Sex, drugs, and bitcoin: How much illegal activity is financed through cryptocurrencies? Rev. Financial Stud. 32 (5), $1798-1853$.

Fry, J., Cheah, E.-T., 2016. Negative bubbles and shocks in cryptocurrency markets. Int. Rev. Financial Analy. 47, $343-352$.

Gerritsen, D.F., Bouri, E., Ramezanifar, E., Roubaud, D., 2020. The profitability of technical trading rules in the Bitcoin market. Finance Res. Lett. 34, 101263. https:// doi.org/10.1016/j.frl.2019.08.011.

Goldfeder, S., Kalodner, H., Reisman, D., Narayanan, A., 2018. When the cookie meets the blockchain: Privacy risks of web payments via cryptocurrencies. Proc. Privacy Enhancing Technol. 2018 (4), 179-199.

Granger, C.J., 1986. Developments in the study of cointegrated economic variables. Oxford Bull. Econ. Stat. 48 (3), $213-228$.

Grobys, K., Ahmed, S., Sapkota, N., 2020. Technical Trading Rules in the Cryptocurrency Market. Finance Res. Lett. 32, 101396. https://doi.org/10.1016/j. frl.2019.101396.

Haug, A.A., MacKinnon, J.G., Michelis, L., 2000. European Monetary Union: a cointegration analysis. J. Int. Money Finance 19 (3), $419-432$.

Jensen, M.C., 1978. Some anomalous evidence regarding market efficiency. J. Financ. Econ. 6 (2-3), 95-101.

Jeon, B.N., Seo, B., 2003. The impact of the Asian financial crisis on foreign exchange market efficiency: The case of East Asian countries. Pacific-Basin Finance J. 11 (4), 509-525.

Johansen, S., 1994. The role of the constant and linear terms in cointegration analysis of nonstationary variables. Econometric Rev. 13 (2), $205-229$. 
Johansen, S., 1991. Estimation and hypothesis testing of cointegration vectors in Gaussian vector autoregressive models. Econometrica: J. Econometric Soc., pp.15511580.

Johansen, S., 1992a. Determination of cointegration rank in the presence of a linear trend. Oxford Bull. Econ. Stat. 54 (3), $383-397$.

Johansen, S., 1992b. Testing weak exogeneity and the order of cointegration in UK money demand data. J. Policy Model. 14 (3), $313-334$.

Johansen, S., 1995. Likelihood-based inference in cointegrated vector autoregressive models. Oxford University Press on Demand.

Kethineni, S., Cao, Y., 2020. The Rise in Popularity of Cryptocurrency and Associate Criminal Activity. International Criminal Justice Review 30 (3), 325-344.

Kus Khalilov, M.C., Levi, A., 2018. A survey on anonymity and privacy in bitcoin-like digital cash systems. IEEE Commun. Surv. Tutorials 20 (3), $2543-2585$.

Kopp, H., Mödinger, D., Hauck, F., Kargl, F., Bösch, C., 2017, April. Design of a privacy-preserving decentralized file storage with financial incentives. In 2017 IEEE European Symposium on Security and Privacy Workshops (EuroS\&PW) (pp. 14-22). IEEE.

Kumar, A., Fischer, C., Tople, S., Saxena, P., 2017, September. A traceability analysis of monero's blockchain. In European Symposium on Research in Computer Security (pp. 153-173). Springer, Cham.

Lo, A.W., Mamaysky, H., Wang, J., 2000. Foundations of technical analysis: Computational algorithms, statistical inference, and empirical implementation. J. Finance 55 (4), 1705-1765.

Liu, J., Li, W., Karame, G.O., Asokan, N., 2018. Toward Fairness of Cryptocurrency Payments. IEEE Secur. Priv. 16 (3), 81-89.

Lütkepohl, H., Krätzig, M., Phillips, P.C. (Eds.), 2004. Applied time series econometrics. Cambridge university press.

Möser, M., Soska, K., Heilman, E., Lee, K., Heffan, H., Srivastava, S., Hogan, K., Hennessey, J., Miller, A., Narayanan, A. and Christin, N., 2017. An empirical analysis of traceability in the monero blockchain. arXiv preprint arXiv:1704.04299.

Miller, N., Yang, Y., Sun, B., Zhang, G., 2019. Identification of technical analysis patterns with smoothing splines for bitcoin prices. J. Appl. Statist. 46 (12), 2289-2297.

Nadarajah, S., Chu, J., 2017. On the inefficiency of Bitcoin. Econ. Lett. 150, 6-9.

Norrbin, S.C., 1996. Bivariate cointegration among European monetary system exchange rates. Appl. Econ. 28 (12), $1505-1513$.

Osterrieder, J., Lorenz, J., 2017. A statistical risk assessment of Bitcoin and its extreme tail behavior. Annals of Financial Economics 12 (01), 1750003.

Phengpis, C., 2006. Market efficiency and cointegration of spot exchange rates during periods of economic turmoil: Another look at European and Asian currency crises. J. Econ. Bus. 58 (4), 323-342.

Rangvid, J., Sørensen, C., 2002. Convergence in the ERM and declining numbers of common stochastic trends. Journal of Emerging Market Finance 1 (2), $183-213$. Richards, A.J., 1995. Comovements in national stock market returns: Evidence of predictability, but not cointegration. J. Monetary Econ. 36 (3), $631-654$.

Ouaddah, A., Elkalam, A.A., Ouahman, A.A., 2017. Towards a novel privacy-preserving access control model based on blockchain technology in IoT. In Europe and MENA Cooperation Advances in Information and Communication Technologies(pp. 523-533). Springer, Cham.

Sensoy, A., 2019. The inefficiency of Bitcoin revisited: A high-frequency analysis with alternative currencies. Finance Res. Lett. 28 , 68-73.

Urquhart, A., 2016. The inefficiency of Bitcoin. Econ. Lett. 148, 80-82.

Vidal-Tomás, D., Ibañez, A., 2018. Semi-strong efficiency of Bitcoin. Finance Res. Lett. 27, 259-265.

Woo, K.Y., 1999. Cointegration analysis of the intensity of the ERM currencies under the European Monetary System. Journal of International Financial Markets, Institutions and Money 9 (4), 393-405.

Niranjan Sapkota completed his Master's degree in Finance from the University of Oulu, Finland with an excellent grade in 2015. He is now working as a Ph.D. student in the Accounting and Finance department with Finance as a major at the University of Vaasa, Finland. His primary focus area is Digital Finance, Financial Uncertainty and Risk. He is also interested in the area of Market Microstructure and High Frequency Trading.

Klaus Grobys studied Economics and Econometrics at the University of Kiel and the University of Stockholm. He holds a Ph.D. degree in Finance passed with distinction from the University of Vasa where he graduated in December 2014. Since January 2015 he works as Assistant Professor of Finance at the University of Vaasa. He has published about 20 articles related to Asset Pricing in journals such as Quantitative Finance, International Review of Financial Analysis, and Journal of Futures Markets among others. His most recent research interests are related to cryptocurrencies. 